\title{
The Validity of the Residuals Approach to Measuring Resilience to Adverse Childhood Experiences
}

Stephanie Cahill ( $\sim$ stephanie.cahill@manchester.ac.uk)

University of Manchester https://orcid.org/0000-0002-2075-5510

Reinmar Hager

University of Manchester School of Biological Science: The University of Manchester Faculty of Biology Medicine and Health

Tarani Chandola

The University of Manchester

\section{Research article}

Keywords: Resilience, residuals, measurement approaches, adverse childhood experiences, ALSPAC

Posted Date: December 6th, 2021

DOI: https://doi.org/10.21203/rs.3.rs-1131709/v1

License: () (i) This work is licensed under a Creative Commons Attribution 4.0 International License. Read Full License

Version of Record: A version of this preprint was published at Child and Adolescent Psychiatry and Mental Health on March 1st, 2022. See the published version at https://doi.org/10.1186/s13034-022-00449-y. 


\section{Abstract}

Background: Resilience is broadly defined as the ability to maintain or regain functioning in the face of adversity. Recent work to harmonise the quantification and definition of resilience quantifies resilience as the residual variance in psychosocial functioning that remains after accounting for adversity exposure. However, there have been no published studies that have formally investigated the validity of this approach. Considering this, we examine the construct and predictive validity of the residuals approach using participants from ALSPAC.

Methods: We regressed exposures of adolescent adversity on adolescent psychopathology scores using the Strength and Difficulties Questionnaire and obtained the residual variance. We investigated construct validity by analysing whether previously identified demographic and resilience factors significantly predicted resilience. Predictive validity of resilience was investigated by comparing the predictive power of resilience with other determinants of psychosocial functioning on two developmental outcomes: depressive symptoms at 18 years, measured by the Short Moods and Feelings Questionnaire, and NEET (Not in Employment, Education or Training) status at 17 and 23 years. The associations between depressive symptoms at 18, resilience, ACEs and covariates were tested using multiple linear regression. NEET status at 17 and 23 were run as separate binary multiple logistic regression models to test associations with resilience and known demographics previously associated with NEET status.

Results: Seven previously identified protective factors, including self-esteem, positive sibling relationship, temperament, and positive perception of school, significantly predicted resilience to adolescent psychopathology, thus providing strong construct validity. Resilience significantly predicted a reduction in depressive symptoms at 18 years, and significantly decreased the likelihood of having NEET status at both 17 years and 23 years, even after taking into account early childhood adversity and other risk factors. None of the socioeconomic factors were significantly associated with resilience.

Conclusions: Our study demonstrates that the residuals method of operationalising resilience has good construct and predictive validity yet recommend replication studies. It has the potential to advance research into the mechanisms and modifiability of resilience.

Trial Registration: Not applicable

\section{Background}

Some individuals do not develop stress-related disorders despite exposure to childhood adversity. This 'resilience' is a well-recognised phenomenon, yet there is considerable variation in the way resilience is defined, operationalised and measured in the literature. An early perspective in resilience research identified resilience as a characteristic or trait of the individual (e.g. (Schultze-Lutter et al., 2016; Tugade \& Fredrickson, 2004)) and considered resilient children to be exceptional individuals (Anthony \& Cohler, 1987). However, this static definition of resilience limits the possibility that resilience can vary across situations and develop through the lifecourse. An emerging consensus in the field is that resilience refers to the dynamic process of adaptation to adversity across the life span, dependent upon context and resources, which enables the individual to successfully negotiate adversity (Cicchetti \& Blender, 2006; Kalisch et al., 2017; Rutter, 2012). The process of resilience is thus central to normal development resulting from an individual's interactions with a range of environmental factors (Bonanno, 2005; Masten, 2014). This definition also assumes that resilience can only be measured after exposure to adversity, as opposed to viewing resilience as a purely intrinsic trait.

Given the negative impact of adverse childhood experiences (ACEs) on a broad range of different physical and mental health aspects (Felitti et al., 1998; Hughes et al., 2017), resilient functioning after exposure to ACEs should be inferred and measured from functioning across different social, emotional, cognitive and/or behavioural domains. By considering the range in severity of ACEs, resilient functioning refers to better psychosocial functioning compared to others with a similar degree of exposure to ACEs. For example, two individuals could have a similar level of moderate functioning, but the individual with a severe history of exposure to ACEs would have a higher level of resilience, when compared to an individual with moderate or low level exposure to ACEs (loannidis et al., 2020).

\section{Residuals Approach to Measuring Resilience}

The "residuals" approach to measuring resilience is an emerging framework that quantifies resilient functioning as doing better than expected given the degree of ACE exposure. Here, the residual scores of the regression models reflect individual degrees of resilient functioning across different domains, taking into account ACE exposure. Thus, resilience is conceptualised as the extent to which an individual is functioning better than expected given their level of adversity exposure (see (Amstadter et al., 2014; Bowes et al., 2010; Collishaw et al., 2016; Cosco et al., 2018; de Vries et al., 2021; Miller-Lewis et al., 2013; Sapouna \& Wolke, 2013; van Harmelen et al., 2017) for a similar approach, with extensive discussion of this method in (loannidis et al., 2020)).

The conceptual basis for the residuals approach rests on the assumption that psychosocial functioning is determined by multiple factors, and that the independent contributions of these determinants can be estimated. One key determinant is exposure to ACEs. Measurements of exposure to adversity are sensitive to the timing, chronicity and severity of ACEs. Yet, substantial variance in psychosocial functioning outcomes remains often unexplained by ACEs. Resilient functioning after adversity is facilitated by protective resilience factors (RFs) that support individuals to adapt and recover from ACEs (Fritz et al., 2018). The wider literature suggests that RFs at the individual, family and community level are associated with a reduced likelihood of developing psychosocial problems (Crush et al., 2018; Fritz et al., 2018; Schaefer et al., 2018).

Our residuals approach to measuring resilience in this study involves an empirical decomposition of variance in an outcome variable (ratings of child psychopathology from the Strength and Difficulties questionnaire (SDQ) (Goodman, 1997), in this case), into components explained by measures of adolescent ACE exposure and a component that is independent of these ACE exposures. The latter residual component captures individual differences in resilience that are not explained by measured variables and provides our putative measure of resilience. That is, individuals with high scores on this component perform better than expected and those with low scores perform worse than expected, corresponding to previously defined resilience and 
vulnerability. It is a measure of the current resilience of an individual, where resilient functioning refers to better mental wellbeing compared to other individuals with similar ACE exposures.

This approach, in a sense, defines resilience as the sum of unmeasured sources of variance in psychosocial functioning, which is not explained by ACE exposure. A limitation of this approach is that the derived measure of resilience will be influenced by the specific variables used and, consequently, different measures of resilience are possible for a specific person at a specific time. However, we are not primarily interested in deriving a singular measure of resilience, but rather in establishing whether the residuals approach provides a useful measure for operationalising resilience. In order to address this, we need to gather evidence to support the use and interpretation of this measure of resilience.

The residuals approach provides a relatively simple way of measuring resilience in relation to ACEs and psychosocial functioning, quantifying resilience on a continuum of resilience to vulnerability. However, the validity of this methodology has not yet been examined.

\section{Construct and Predictive Validity}

Resilience is an unobservable, hypothetical construct. The challenge lies in how to measure resilience in a robust way that enables significant advances in the field. Cronbach and Meehl's (1955) seminal article, "Construct Validity in Psychological Tests", argued that there must be evidence that a measure of a given construct relates to measures of other constructs in a theoretically predictable way (Cronbach \& Meehl, 1955). Validity analyses are common approaches to assessing psychometric quality (Zumbo \& Chan, 2014) and quantifying a measure's predictive ability for a key outcome or association with a theoretically relevant construct can provide further support. Predictive validity of resilience must show predictive correlations with later positive psychosocial outcomes.

In our study, we first investigated the construct validity of resilience, when measured using the residuals approach, by analysing whether previously identified RFs and demographic factors significantly predict resilience as measured by the residuals approach. Specifically, we hypothesize that previously theorised resilience factors and associated demographic variables in childhood significantly predict resilience to SDQ total difficulties at 16 years of age.

Second, predictive validity was assessed by comparing the predictive power of the residuals measure of resilience with other determinants of psychosocial functioning on two developmental outcomes. The first, proximal, outcome is a measure of depressive symptoms at age 18, as measured by the Short Mood and Feelings Questionnaire (SMFQ) (Angold et al., 1995). The SMFQ is designed to examine the presence of depressive symptoms via a 13-item self-reported questionnaire and is well validated for epidemiological studies (Turner et al., 2014). We predict that resilience to SDQ difficulties at 16 years significantly decreases the risk of depressive symptoms at 18 years.

The second, distal, outcome is NEET (Not in Employment, Education or Training) status at 17 and 23 years. NEET status demonstrates an objective and ecologically valid measure of poor functioning (Organisation for Economic Co-operation and Development, 2012). Here, we predict that resilience to SDQ difficulties at 16 years significantly reduces the risk of having NEET status at 17 years and 23 years.

\section{Method}

\section{Participants}

The sample comprised 15,454 participants from the Avon Longitudinal Study of Parents and Children (ALSPAC), a multigenerational, longitudinal cohort study that recruited pregnant women resident in the former Avon Health Authority in south-west England who had an estimated due date between 1 st April 1991 and 31st December 1992 (Boyd et al., 2013; Fraser et al., 2012). After data cleaning, our sample comprised of 14,694 participants. The study website with detailed information about ALSPAC is available at (http://www.bristol.ac.uk/alspac/), which includes a fully searchable data dictionary and variable search tool (http://www.bristol.ac.uk/alspac/researchers/access/).

\section{Exposure to Adverse Childhood Experiences (ACEs)}

Mothers, partners, and the study child were asked 87 questions about the child's exposure to 15 ACEs between the ages of 11 and 16 years (Table 1 ). These adversities were all prospectively measured from questionnaires with the cohort child's mother, the mother's partner or the cohort child themselves, or collected at four clinic sessions the children attended between 11 and 15.5 years (see supplementary table $x$ for further information on the definitions of each ACE with the timing of collection, type of data collection and number of contributing questions). While there is currently much debate within ACE research about what constitutes 'adversity' and the controversial application of ACE scores used at the individual level to predict ill health (see (Lacey \& Minnis, 2020; Meehan et al., 2021), within the present study ACE constructs were derived as binary measures of exposure as described by Houtepen and colleagues for specific use within the ALSPAC cohort, to encourage replication (Houtepen et al., 2018). 
Table 1

Description of variables and data sources used to derive binary measures of adolescent ACEs.

\begin{tabular}{|c|c|c|}
\hline ACE & Description & Data Source \\
\hline Bullying & $\begin{array}{l}\text { Five variables on frequency someone threatened/blackmailed, told lies } \\
\text { about, put down, upset, peer pressured teenager }\end{array}$ & $\begin{array}{l}\text { Teen Focus Research clinic at } 12.5 \mathrm{yrs} \text { and } 15.5 \mathrm{yrs} \text {. } \\
\text { Child completed questionnaire } 16 \mathrm{yrs}\end{array}$ \\
\hline Emotional Abuse & $\begin{array}{l}\text { Four variables asking if parent or partner has been emotionally cruel to } \\
\text { the study child }\end{array}$ & $\begin{array}{l}\text { Mother and partner completed questionnaires at } \\
11 \mathrm{yrs} 2 \text { months }\end{array}$ \\
\hline Emotional Neglect & $\begin{array}{l}17 \text { variables asking a range of questions on interactions with } \\
\text { parent/carers }\end{array}$ & $\begin{array}{l}\text { Teen Focus Research clinic at } 12.5 \mathrm{yrs}, 13.5 \mathrm{yrs} \text { and } \\
15.5 y \text { rs. Child based and child completed } \\
\text { questionnaires } 16 y \text { rs }\end{array}$ \\
\hline Financial Difficulties & Six variables on homelessness and inability to pay for food/heating & $\begin{array}{l}\text { Mother and partner completed questionnaires at } \\
11 \mathrm{yrs} 2 \text { months }\end{array}$ \\
\hline $\begin{array}{l}\text { Parent Mental Health } \\
\text { Problems or Suicide } \\
\text { Attempt }\end{array}$ & $\begin{array}{l}14 \text { variables on diagnosed mental health problems, suicide attempts, } \\
\text { self harm, and medication for anxiety or depression }\end{array}$ & $\begin{array}{l}\text { Mother, partner and child completed questionnaires } \\
\text { at } 11 \mathrm{yrs} 2 \mathrm{~m}, 12 \mathrm{yrs} 1 \mathrm{~m} \text { and } 16 \mathrm{yrs}\end{array}$ \\
\hline $\begin{array}{l}\text { Neighbourhood } \\
\text { Satisfaction }\end{array}$ & Four variables of neighbourhood satisfaction & $\begin{array}{l}\text { Teen Focus research clinic at } 15.5 \mathrm{yrs} \text { and child } \\
\text { completed questionnaire at } 14 \mathrm{yrs}\end{array}$ \\
\hline Parent Child Bond & $\begin{array}{l}\text { Three variables measuring how close the partner or mother feels to the } \\
\text { study child and a clinic-based measurement of adult/child harmonious } \\
\text { interactions }\end{array}$ & $\begin{array}{l}\text { Teen Focus research clinic at } 12.5 \mathrm{yrs} \text {. Mother and } \\
\text { partner completed questionnaires at } 12 \mathrm{yrs} 1 \mathrm{~m}\end{array}$ \\
\hline $\begin{array}{l}\text { Parent Convicted } \\
\text { Offence }\end{array}$ & Four variables on whether parent or carer convicted of offence & $\begin{array}{l}\text { Mother and partner completed questionnaires at } \\
11 \mathrm{yrs} 2 \mathrm{~m} \text { and } 12 \mathrm{yrs} 1 \mathrm{~m}\end{array}$ \\
\hline Parental Separation & Six variables on whether parents had divorced or separated & $\begin{array}{l}\text { Mother, partner, and child completed questionnaires } \\
\text { at } 11 \mathrm{yrs} 2 \mathrm{~m} \text { and } 16 \mathrm{yrs}\end{array}$ \\
\hline Physical Abuse & $\begin{array}{l}\text { Four variables asking if parent or partner has been physically cruel to } \\
\text { the study child }\end{array}$ & $\begin{array}{l}\text { Mother and partner completed questionnaires at } \\
11 \mathrm{yrs} 2 \text { months }\end{array}$ \\
\hline Social Support - Child & $\begin{array}{l}\text { Eight variables on number of close friends and satisfaction with } \\
\text { friendships }\end{array}$ & $\begin{array}{l}\text { Teen Focus Research clinic at } 12.5 y r s, 13.5 y r s \text { and } \\
15.5 y r s .\end{array}$ \\
\hline $\begin{array}{l}\text { Social Support - } \\
\text { Parent }\end{array}$ & Two variables on whether the parent has anybody to share feelings with & $\begin{array}{l}\text { Mother and partner completed questionnaires at } \\
12 \mathrm{yrs} 1 \mathrm{~m}\end{array}$ \\
\hline $\begin{array}{l}\text { Household Substance } \\
\text { Abuse }\end{array}$ & One variable asking if respondent has ever had a drug addiction & Partner completed questionnaire at 11yrs $2 \mathrm{~m}$ \\
\hline $\begin{array}{l}\text { Violence Between } \\
\text { Child and Partner }\end{array}$ & Six variables on violence in a romantic relationship & Teen Focus research clinic at $13.5 y$ rs \\
\hline $\begin{array}{l}\text { Violence Between } \\
\text { Parents }\end{array}$ & Two variables on physical cruelty between parents & $\begin{array}{l}\text { Mother and partner completed questionnaires at } \\
11 \mathrm{yrs} 2 \mathrm{~m}\end{array}$ \\
\hline
\end{tabular}

\section{Strength and Difficulties Questionnaire}

The SDQ is one of the most commonly used ratings of child psychopathology in epidemiological studies (Goodman, 1997). The SDQ questionnaire was selfreported at 16 years 6 months and comprises 20 items relating to four different psychosocial scales: emotional symptoms; conduct problems; hyperactivity/inattention and peer problems. Responses are scored using a three-point Likert scale and the answer summed to give a total difficulties score out of 40 .

\section{Short Mood and Feelings Questionnaire}

The SMFQ was self-reported by the study child at 18 years 6 months. The SMFQ is designed to examine the presence of depressive symptoms via a 13 -item self-reported questionnaire and is well validated (Turner et al., 2014). As each question is scored between $0-2$, the resulting summary score can range between 0-26 with higher scores being more indicative of higher depression. The SMFQ has shown good validity and reliability for scores of 11 or more determining the presence of depression (Angold et al., 2002).

\section{Resilience}

In order to investigate the validity of the residuals approach, we regressed binary exposures of ACEs experienced in adolescence (11-16yrs) on the total difficulties score of the SDQ at 16 years 6 months. We extracted the residuals scores as these reflect a spectrum ranging from risk to resilient functioning, i.e., the extent to which an individual has better, or worse, SDQ outcomes than the average score expected given their exposure to ACEs over adolescence.

\section{NEET Status at 17 and 23}

NEET was assessed using the same self-report questionnaire at 17 and 23 years, which included two questions on whether the participants were currently enrolled in any education/training programme or employed. A binary variable was created, which classified the young person as NEET if the answer to both questions was negative, in line with the definition used by the Office for National Statistics (ONS, 2018). 


\section{Resilience Factors}

Potential individual, family and community factors associated with resilience were chosen based on previous literature (Johnson \& Wiechelt, 2004; Khambati et al., 2018). At the individual level these include higher IQ (Harpur et al., 2015; Pargas et al., 2010), an easy temperament (Martinez-Torteya et al., 2009), internal locus of control (Bolger \& Patterson, 2001), high mental flexibility (Qouta et al., 2001), high self-esteem (Pargas et al., 2010), high linguistic ability (Werner \& Smith, 2001) and high cognitive skills (Bennett et al., 2005; Herrenkohl et al., 1995). At the family level these include attachment to grandparent (Werner, 1997), maternal parenting (Herrenkohl et al., 1995; Schechter et al., 2017) and sibling relationship (Khambati et al., 2018). At the community level these include high school engagement (Herrenkohl et al., 2005; Williams \& Nelson-Gardell, 2012), positive perception of school (Perkins \& Jones, 2004), engagement with religion (Herrenkohl et al., 2005; Perkins \& Jones, 2004), regular engagement in extracurricular activity (Peck et al., 2008) and supportive friendships (Graber et al., 2016; Haskett et al., 2006). See Supplementary Table 1 for a full description of how each resilience factor was derived, including methods and times of measurement.

\section{Covariates}

The analyses were adjusted for relevant demographic, socioeconomic, lifestyle and health variables. These included sex of the child (dichotomous variable); maternal age at birth; birthweight; gestation; maternal smoking in the 2nd trimester of pregnancy (binary yes/no); parity, defined as the number of times that the woman had given birth to a child with a gestational age of 24 weeks or more; ethnicity of the child (coded white/BAME); socioeconomic status based on maternal and partner educational attainment (none/Certificate of Standard Education to University degree); occupational social class as classified by the Office of Population Censuses and Surveys in 1990 (classes I (professional/managerial) to V (unskilled/manual workers)); home ownership at birth (binary rented/owned); marital status at birth (binary married/not married); mother BMI category pre-pregnancy (underweight, normal weight, overweight, obese); and Alcohol Use Disorders Identification Test (AUDIT) measured when the child was 17.5 years (continuous score with higher score indicating higher risk of problem drinking).

\section{Missing Data}

Missing data on all ACE items (outcome variables and covariates) were estimated using multiple imputation by chained equations (MI). The proportion of missingness in the analytic sample ranged from $0-78.1 \%$. Mothering score and nicotine dependency scores were removed from the analysis due to too high missingness (>80\%). All variables except ID number were used as predictors in the imputation models. Ml estimates missing information under the Missing at Random (MAR) assumption (Little \& Rubin, 2019). If all variables associated with the missing data generation processes are included in the imputation models then missing values can be reliably imputed (Ploubidis et al., 2014). ACE measures were computed for participants who answered at least $50 \%$ of the questions used to derive the binary measures of ACEs (Houtepen et al., 2018). These participants have higher socioeconomic status than the full cohort and including only these participants will lead to lower estimates of ACE occurrence and could result in selection bias (Howe et al., 2013). To make the xx assumption more plausible, we included sociodemographic indicators that are associated with missingness, many of the ACEs, NEET status and SDQ outcomes (mother's home ownership status at birth, mother and partner's highest educational qualification, maternal age at birth, maternal marital status at birth, birthweight, parity, gestational age, maternal BMI, maternal smoking during pregnancy, alcohol dependency, ethnicity of the child). Twenty imputed datasets were created using the mice (Multivariate Imputation by Chained Equations) package version 3.11.0 in R-4.0.3 with 20 iterations per dataset (Buuren \& Groothuis-Oudshoorn, 2010). A comparison of observed and imputed data (Table 1) suggests the imputation procedure was conducted consistent to expected missingness patterns. Reflected by a higher missingness rate in more deprived participants (Howe et al., 2013), disadvantaged sociodemographic indicators (manual social class, low parental education, and rented home tenure) were lower in the original data than the imputed data. (Table 1). ACE exposure estimates are higher in the imputed data, as expected.

In the imputed data, emotional neglect was the most common ACE (19.0\%), which is in line with previous reported prevalence of emotional neglect as ACE exposure in the UK population (Bellis et al., 2013). Parent convicted of an offence had the lowest prevalence (2.5\%), which is lower than other ACE studies in the UK (4.0 to 4.1\%) (Bellis et al., 2013; Hughes et al., 2016) and the US (7\%) (Crouch et al., 2019) but still in line with parental criminal estimates in highincome countries (Kessler et al., 2010). NEET status at 17 years and 23 years are both higher in the imputed data (but in line with UK estimates for NEET status in the UK in 16 - 18 year olds in 2007), and slightly lower than reported NEET status for 23 year olds in the UK in 2013 (in the corresponding year to data collection in ALSPAC) (Department for Education, 2013).

\section{Statistical Analyses}

All analyses were performed in R version 4.0.3 (2020-10-10), RStudio version 1.3.1093 for Windows. SMFQ and SDQ were both positively skewed (SMFQ skewness $=1.22$; SDQ skewness $=1.28$ ) so were square-root transformed to reduce non-normality of the raw data distributions, and meet the assumptions of the predictive linear model (See supplementary figure 1). Resilience was derived from the standardised residuals of a multiple linear regression model where binary exposures of ACEs experienced in adolescence (11-16yrs) were regressed on the total difficulties score of the SDQ at 16 years 6 months. The associations between resilience, resilience factors and covariates, and resilience and NEET status and SMFQ outcomes were modelled in separate models. The associations between depressive symptoms at 18, resilience, ACEs and covariates were tested using multiple linear regression. NEET status at 17 and 23 were run as separate binary multiple logistic regression models to test associations with resilience and known demographics previously associated with NEET status. All reported results are from the pooled estimates from the multiple imputed analyses. The standardized regression coefficients reported in the results section resulted from rerunning the final models in the combined dataset containing all the imputed datasets.

\section{Results}


The characteristics of the study sample are summarised in Table 2 and include the original and imputed data. $47.7 \%$ of the children were female, with an overwhelming majority of the children being white (97.5\%). The majority of the mothers were married (75.8\%) and owned their own home at birth (77\%). While the prevalence of individual ACEs in the initial sample ranged from $0.1 \%$ (substance abuse in the household) to $15.2 \%$ (emotional neglect), in the imputed sample the range was from $2.5 \%$ (parent convicted of an offence) to $19.0 \%$ (emotional neglect). 
Table 2

Prevalence estimates and sample characteristics for ACE, SDQ, NEET status and demographic measures in the original ALSPAC data and imputed data.

\begin{tabular}{|c|c|c|c|}
\hline \multirow[t]{2}{*}{ Variable } & \multicolumn{2}{|c|}{ Original Data } & \multirow{2}{*}{$\begin{array}{l}\text { Imputed data }(\mathrm{N}=\mathbf{2 9 3 8 8 0}) \\
\text { Mean (sd) for continuous variables. \% for } \\
\text { categorical variables }\end{array}$} \\
\hline & $\mathbf{N}$ & $\begin{array}{l}\text { Mean (sd) for continuous variables, } \% \text { for } \\
\text { categorical variables }\end{array}$ & \\
\hline SDQ Total Difficulties (16yrs) & 5566 & $6.1(4.8)$ & $6.8(5.3)$ \\
\hline Physical Abuse & 7662 & 0.4 & 3.2 \\
\hline Emotional Abuse & 7655 & 2.1 & 5.7 \\
\hline Emotional Neglect & 5728 & 15.2 & 19.0 \\
\hline Bullying & 6841 & 14.5 & 15.5 \\
\hline Violence Between Parents & 7675 & 0.8 & 4.0 \\
\hline Substance Abuse Household & 3618 & 0.1 & 6.9 \\
\hline Parental Mental Health Problems & 3652 & 1.9 & 10.2 \\
\hline Parent Convicted Offence & 6864 & 0.7 & 2.5 \\
\hline Parental Separation & 5567 & 8.3 & 13.2 \\
\hline Financial Difficulties & 7605 & 0.9 & 3.3 \\
\hline Neighbourhood Satisfaction & 5287 & 9.8 & 12.7 \\
\hline Social Support Child & 6639 & 5.2 & 7.3 \\
\hline Social Support Parent & 6948 & 2.3 & 4.1 \\
\hline $\begin{array}{l}\text { Violence Between Child and } \\
\text { Partner }\end{array}$ & 3977 & 11.0 & 14.2 \\
\hline Parent Child Bond & 5378 & 5.6 & 9.0 \\
\hline Gestation (weeks) & 13788 & $39.4(1.9)$ & $39.4(1.9)$ \\
\hline $\begin{array}{l}\text { Social Class Household ( } 32 \mathrm{wk} \\
\text { gestation) }\end{array}$ & 8773 & & \\
\hline I-Professional & & 11.3 & 9.9 \\
\hline II - Managerial and Technical & & 44.4 & 40.7 \\
\hline IIIM - Skilled Manual & & 11.7 & 14.5 \\
\hline IIINM Skilled Non-Manual & & 30.2 & 29.5 \\
\hline IV - Partly Skilled & & 2.2 & 4.1 \\
\hline V-Unskilled & & 0.2 & 1.3 \\
\hline Maternal Smoking 2nd Trimester & 12984 & & \\
\hline No & & 80.2 & 79.5 \\
\hline Yes & & 19.8 & 20.5 \\
\hline IQ Category & 7421 & & \\
\hline Exceptionally Low & & 1.8 & 3.6 \\
\hline Low & & 5.3 & 7.4 \\
\hline Low Average & & 12.1 & 13.8 \\
\hline Average & & 43.8 & 42.2 \\
\hline High Average & & 19.5 & 16.8 \\
\hline High & & 9.3 & 8.5 \\
\hline Exceptionally High & & 8.1 & 7.7 \\
\hline Reading Speed (reading age) & 6823 & $10.4(1.7)$ & $10.2(1.8)$ \\
\hline Reading Accuracy (reading age) & 6840 & $9.9(1.8)$ & $9.7(1.8)$ \\
\hline $\begin{array}{l}\text { Reading Comprehension (reading } \\
\text { age) }\end{array}$ & 6840 & $9.6(1.7)$ & $9.4(1.8)$ \\
\hline
\end{tabular}




\begin{tabular}{|c|c|c|c|}
\hline \multirow[t]{2}{*}{ Variable } & \multicolumn{2}{|c|}{ Original Data } & \multirow{2}{*}{$\begin{array}{l}\text { Imputed data }(\mathrm{N}=293880) \\
\text { Mean (sd) for continuous variables. \% for } \\
\text { categorical variables }\end{array}$} \\
\hline & $\mathbf{N}$ & $\begin{array}{l}\text { Mean (sd) for continuous variables, \% for } \\
\text { categorical variables }\end{array}$ & \\
\hline Self Esteem: Global Self-Worth & 6833 & $19.2(3.4)$ & $19.1(3.5)$ \\
\hline $\begin{array}{l}\text { Self Esteem: Scholastic } \\
\text { Competence }\end{array}$ & 6844 & $17.0(3.7)$ & $16.9(3.7)$ \\
\hline Locus of Control & 7038 & & \\
\hline Externalised & & 57.3 & 60.0 \\
\hline Internalised & & 42.7 & 40.0 \\
\hline $\begin{array}{l}\text { Mental Flexibility (mean reaction } \\
\text { time) }\end{array}$ & 6871 & $598.5(67.6)$ & $602.1(70.7)$ \\
\hline Cognitive Skills & 8360 & $2.0(0.1)$ & $1.9(0.1)$ \\
\hline Temperament & 8393 & $17.3(3.4)$ & $17.2(3.4)$ \\
\hline Grandparent Attachment & 8118 & & \\
\hline Yes & & 45.8 & 46.0 \\
\hline No & & 54.2 & 54.0 \\
\hline Sibling Relationship Score & 6294 & $31.3(5.5)$ & $31.4(5.5)$ \\
\hline School Attendance & 8052 & & \\
\hline Over 10 days absent & & 6.7 & 8.0 \\
\hline Less than 10 days absent & & 93.3 & 92.0 \\
\hline School Perception & 3215 & $23.6(3.9)$ & $23.3(4.1)$ \\
\hline Religion & 8077 & & \\
\hline No & & 44.1 & 47.0 \\
\hline Yes & & 55.9 & 53.0 \\
\hline Extra-Curricular Activities & 5571 & & \\
\hline Yes & & 42.2 & 38.3 \\
\hline No & & 57.8 & 61.7 \\
\hline Friendship & 6650 & $20.9(2.2)$ & $20.8(2.2)$ \\
\hline Birthweight (kg) & 13615 & $3.4(0.6)$ & $3.4(0.6)$ \\
\hline Maternal Age & 13788 & $28.0(5.0)$ & $28.0(5.0)$ \\
\hline Home Ownership Status birth & 12406 & & \\
\hline Owned & & 76.0 & 74.3 \\
\hline Rented & & 24.0 & 25.7 \\
\hline Marital Status Birth & 12915 & & \\
\hline Not Married & & 25.1 & 25.9 \\
\hline Married & & 74.9 & 74.1 \\
\hline Parity & 12757 & & \\
\hline 0 & & 44.8 & 44.3 \\
\hline 1 & & 35.0 & 34.4 \\
\hline 2 & & 14.3 & 14.3 \\
\hline 3 & & 4.2 & 4.5 \\
\hline 4 & & 1.2 & 1.5 \\
\hline $5+$ & & 0.5 & 0.9 \\
\hline $\begin{array}{l}\text { Mother Highest Education } \\
\text { Qualification }\end{array}$ & 12251 & & \\
\hline CSE/None & & 20.2 & 21.3 \\
\hline
\end{tabular}




\begin{tabular}{|c|c|c|c|}
\hline \multirow[t]{2}{*}{ Variable } & \multicolumn{2}{|c|}{ Original Data } & \multirow{2}{*}{$\begin{array}{l}\text { Imputed data }(\mathrm{N}=\mathbf{2 9 3 8 8 0}) \\
\text { Mean (sd) for continuous variables. \% for } \\
\text { categorical variables }\end{array}$} \\
\hline & $\mathbf{N}$ & $\begin{array}{l}\text { Mean (sd) for continuous variables, \% for } \\
\text { categorical variables }\end{array}$ & \\
\hline Vocational & & 9.9 & 10.0 \\
\hline O Level & & 34.6 & 34.2 \\
\hline A Level & & 22.5 & 21.9 \\
\hline Degree & & 12.9 & 12.6 \\
\hline Ethnicity & 12163 & & \\
\hline White & & 97.4 & 97.1 \\
\hline BAME & & 2.6 & 2.9 \\
\hline Matemal BMI Category & 11378 & & \\
\hline Underweight & & 5.0 & 5.4 \\
\hline Normal Weight & & 73.4 & 72.8 \\
\hline Overweight & & 16.1 & 16.1 \\
\hline Obese & & 5.5 & 5.7 \\
\hline $\begin{array}{l}\text { Partner Highest Education } \\
\text { Qualification }\end{array}$ & 11775 & & \\
\hline CSE/None & & 26.1 & 27.9 \\
\hline Vocational & & 8.5 & 8.8 \\
\hline O Level & & 22.6 & 20.9 \\
\hline A Level & & 26.0 & 25.2 \\
\hline Degree & & 18.2 & 17.3 \\
\hline Sex & 14694 & & \\
\hline Male & & 51.0 & 51.0 \\
\hline Female & & 49.0 & 49.0 \\
\hline $\begin{array}{l}\text { SMFQ - Depressive Symptoms } \\
\text { (18yrs) }\end{array}$ & 3305 & $6.8(5.9)$ & $7.1(6.2)$ \\
\hline NEET at $17 \mathrm{yrs}$ & 4052 & & \\
\hline Yes & & 4.3 & 9.3 \\
\hline No & & 95.7 & 90.7 \\
\hline NEET at 23 yrs & 3997 & & \\
\hline Yes & & 7.0 & 11.0 \\
\hline No & & 93.0 & 89.0 \\
\hline AUDIT Score at $17 y r s$ & 4108 & $7.0(4.9)$ & $7.2(5.1)$ \\
\hline
\end{tabular}

\section{Deriving resilience from the residuals of SDQ total difficulties score predicted by ACEs}

Eight of the fifteen ACEs were significantly associated with SDQ total behavioural difficulties at 16 years of age. Financial difficulties, emotional abuse, and lack of parent child bond were the strongest predictors of total behavioural difficulties (see Table 3). Resilience was derived using the residuals from the full model $($ mean $=-0.99, \mathrm{sd}=5.00$, range $=-25.34-17.63)$. See figure 1 for residual distribution of the multiple linear regression model. 
Table 3

Adolescent ACEs predicting Total Behavioural Difficulties on Strength and Difficulties Questionnaire. Adjusted $\mathrm{R}^{2}=0.091(0.066-$ $0.119)$

\begin{tabular}{|c|c|c|c|c|c|c|}
\hline \multirow[b]{2}{*}{ Predictors } & \multicolumn{6}{|c|}{ SDQ Total Behavioural Difficulties Adolescent ACEs } \\
\hline & Estimates & std. Beta & standardized $\mathrm{Cl}$ & $\mathrm{Cl}$ & $t$ value & $p$ \\
\hline Intercept & 2.15 & 0.94 & $0.93-0.94$ & $2.12-2.19$ & 121.56 & $<0.001$ \\
\hline Physical Abuse (11-16yrs) & -0.02 & -0.01 & $-0.02-0.00$ & $-0.44-0.39$ & -0.11 & 0.912 \\
\hline Emotional Abuse (11-16yrs) & 0.30 & 0.13 & $0.12-0.14$ & $0.06-0.55$ & 2.56 & 0.016 \\
\hline Emotional Neglect (11-16yrs) & 0.17 & 0.07 & $0.07-0.08$ & $0.08-0.26$ & 3.96 & $<0.001$ \\
\hline Bullying (11-16yrs) & 0.20 & 0.09 & $0.08-0.09$ & $0.12-0.29$ & 4.78 & $<0.001$ \\
\hline Violence Between Parents (11-16yrs) & 0.05 & 0.02 & $0.01-0.03$ & $-0.22-0.32$ & 0.37 & 0.711 \\
\hline Household Substance Abuse (11-16yrs) & 0.11 & 0.05 & $0.04-0.06$ & $-0.22-0.44$ & 0.69 & 0.497 \\
\hline Parental Mental Health Problems (11-16yrs) & 0.18 & 0.08 & $0.07-0.09$ & $-0.01-0.37$ & 1.92 & 0.066 \\
\hline Parent Convicted of Offence (11-16yrs) & 0.03 & 0.01 & $-0.00-0.02$ & $-0.20-0.26$ & 0.27 & 0.789 \\
\hline Parental Separation (11-16yrs) & 0.09 & 0.04 & $0.03-0.04$ & $-0.04-0.23$ & 1.44 & 0.162 \\
\hline Financial Difficulties (11-16yrs) & 0.31 & 0.13 & $0.12-0.14$ & $0.06-0.55$ & 2.57 & 0.016 \\
\hline Neighbourhood Dissatisfaction (11-16yrs) & 0.23 & 0.10 & $0.09-0.10$ & $0.11-0.35$ & 3.85 & 0.001 \\
\hline No Social Support - Child (11-16yrs) & 0.27 & 0.12 & $0.11-0.13$ & $0.12-0.42$ & 3.76 & 0.001 \\
\hline No Social Support - Parent (11-16yrs) & 0.15 & 0.07 & $0.06-0.07$ & $-0.03-0.32$ & 1.68 & 0.103 \\
\hline Violence Between Child and Partner (11-16yrs) & 0.20 & 0.09 & $0.08-0.09$ & $0.10-0.30$ & 4.00 & $<0.001$ \\
\hline No Parent Child Bond (11-16yrs) & 0.31 & 0.13 & $0.13-0.14$ & $0.17-0.46$ & 4.48 & $<0.001$ \\
\hline
\end{tabular}

\section{Construct Validity - Predicting Resilience with Resilience Factors}

We assessed the construct validity of the residuals approach by investigating whether previously identified RFs significantly predict resilience as measured by the residuals approach. The multiple linear regression model of resilience predicted by resilience factors and demographic variables is given in Table 4 .

Seven of the 17 resilience factors were associated with higher levels of resilience, and their effect on resilience was in line with predictions based on prior research. Somewhat surprisingly, no socioeconomic factors significantly predicted resilience. Parity is the only demographic variable significantly associated with higher levels of resilience. A parity of 1 , i.e. having had just one child, (std. $\beta=0.15, p<0.001$ ) and a parity of 3 (std. $\beta=0.34, p=0.010)$ was associated with higher levels of resilience, compared to a parity of 0 . Having a parity of 2 trends towards a significant association with higher levels of resilience ( $p=$ 0.058)

Individual RFs: High cognitive skills at 6 years, 9 months was the strongest predictor of higher levels of resilience $(\beta=2.75, p=0.004)$. Reading comprehension at 9 years was associated with higher levels of resilience at 16 years $(\beta=0.21, p=0.008)$. Having a less emotional temperament at 5 years 9 months was associated with higher levels of resilience at 16 years $(\beta=0.28, p=<0.001)$. A high degree of global self-worth at 8 years was associated with higher levels of resilience at 16 years $(\beta=0.08, p=0.033)$. Having a very high IQ trended towards a significant positive association $(p=0.066)$ when compared to an exceptionally low IQ in predicting resilience.

Family RFs: The only family resilience factor significantly associated with higher levels of resilience was positive sibling relationship, measured at 11 years, 8 months $(\beta=0.05, p=0.007)$.

Community RFs: A positive opinion of school at 11 years and 14 years was associated with higher levels of resilience at 16 years $(\beta=0.13, p=<0.001)$. Regularly participating in extracurricular activities from $6-16$ years was associated with higher levels of resilience at 16 years $(\beta=0.54, p=<0.001)$ when compared with not participating in extracurricular activities. 
Table 4

Multivariate linear regression model of resilience predicted by resilience factors and demographic variables. Adjusted $R^{2}=0.139$ $(0.116-0.164)$

\begin{tabular}{|c|c|c|c|c|c|}
\hline \multirow[b]{2}{*}{ Predictors } & \multicolumn{5}{|c|}{ Resilience to SDQ Total Difficulties Resilience Factors \& Demographic Variables } \\
\hline & $\beta$ & std. Error & $\mathrm{Cl}$ & $t$ value & $p$ \\
\hline Intercept & -21.85 & 2.49 & $-26.94--16.77$ & -8.77 & $<0.001$ \\
\hline Social Class IV - Partly skilled & 0.20 & 1.14 & $-2.15-2.54$ & 0.17 & 0.864 \\
\hline Social Class IIINM - Skilled non-manual & -0.17 & 0.82 & $-1.86-1.51$ & -0.21 & 0.834 \\
\hline Social Class IIIM - Skilled manual & 0.09 & 0.88 & $-1.71-1.89$ & 0.10 & 0.918 \\
\hline Social Class II - Managerial and technical & 0.10 & 0.83 & $-1.60-1.81$ & 0.13 & 0.901 \\
\hline Social Class I - Professional & 0.40 & 0.81 & $-1.24-2.05$ & 0.50 & 0.620 \\
\hline Maternal Smoking (2nd Trimester) & -0.18 & 0.22 & $-0.62-0.27$ & -0.80 & 0.431 \\
\hline Birthweight (kg) & 0.15 & 0.15 & $-0.15-0.45$ & 0.98 & 0.331 \\
\hline Maternal Age (yrs) & -0.01 & 0.02 & $-0.04-0.02$ & -0.71 & 0.482 \\
\hline Home Ownership Status Birth (Owned) & 0.15 & 0.20 & $-0.26-0.56$ & 0.73 & 0.469 \\
\hline Marital Status Birth (Married) & -0.19 & 0.19 & $-0.57-0.19$ & -1.00 & 0.322 \\
\hline Parity (1) & 0.93 & 0.15 & $0.63-1.23$ & 6.20 & $<0.001$ \\
\hline Parity (2) & 0.53 & 0.27 & $-0.02-1.07$ & 1.97 & 0.058 \\
\hline Parity (3) & 0.92 & 0.34 & $0.23-1.60$ & 2.70 & 0.010 \\
\hline Parity (4) & 1.59 & 0.82 & $-0.09-3.26$ & 1.95 & 0.062 \\
\hline Parity (5+) & 0.60 & 1.12 & $-1.71-2.91$ & 0.54 & 0.596 \\
\hline Mum Highest Education - Vocational & -0.23 & 0.37 & $-1.00-0.53$ & -0.63 & 0.534 \\
\hline Mum Highest Education - O Level & 0.03 & 0.23 & $-0.44-0.50$ & 0.14 & 0.889 \\
\hline Mum Highest Education - A Level & 0.18 & 0.28 & $-0.38-0.75$ & 0.66 & 0.512 \\
\hline Mum Highest Education - Degree & 0.09 & 0.30 & $-0.51-0.69$ & 0.30 & 0.764 \\
\hline Ethnicity (BAME) & 0.57 & 0.53 & $-0.53-1.66$ & 1.06 & 0.297 \\
\hline Maternal BMI (Underweight) & -0.51 & 0.31 & $-1.14-0.12$ & -1.64 & 0.109 \\
\hline Maternal BMI (Overweight) & 0.26 & 0.25 & $-0.24-0.76$ & 1.06 & 0.298 \\
\hline Maternal BMI (Obese) & -0.51 & 0.41 & $-1.36-0.33$ & -1.25 & 0.222 \\
\hline Partner Highest Education - Vocational & 0.08 & 0.33 & $-0.60-0.76$ & 0.24 & 0.815 \\
\hline Partner Highest Education - O Level & 0.02 & 0.27 & $-0.52-0.57$ & 0.08 & 0.937 \\
\hline Partner Highest Education - A Level & -0.11 & 0.27 & $-0.67-0.45$ & -0.40 & 0.689 \\
\hline Partner Highest Education - Degree & -0.04 & 0.32 & $-0.68-0.61$ & -0.11 & 0.912 \\
\hline Gestation (weeks) & -0.04 & 0.04 & $-0.13-0.05$ & -0.90 & 0.373 \\
\hline Sex (Female) & -0.24 & 0.16 & $-0.56-0.07$ & -1.56 & 0.129 \\
\hline Low IQ (70 - 79) & 0.28 & 0.84 & $-1.47-2.03$ & 0.33 & 0.743 \\
\hline Low Average IQ (80-89) & 0.95 & 0.74 & $-0.58-2.49$ & 1.29 & 0.211 \\
\hline Average IQ (90-109) & 1.19 & 0.79 & $-0.44-2.82$ & 1.52 & 0.143 \\
\hline High Average IQ (110-119) & 1.21 & 0.71 & $-0.25-2.67$ & 1.71 & 0.100 \\
\hline Very High IQ (120-129) & 1.47 & 0.76 & $-0.10-3.04$ & 1.92 & 0.066 \\
\hline Exceptionally High IQ (130+) & 1.31 & 0.76 & $-0.25-2.87$ & 1.72 & 0.097 \\
\hline Reading Speed (yrs) & -0.01 & 0.05 & $-0.12-0.10$ & -0.16 & 0.872 \\
\hline Reading Accuracy (yrs) & 0.04 & 0.07 & $-0.10-0.19$ & 0.62 & 0.541 \\
\hline Reading Comprehension (yrs) & 0.21 & 0.07 & $0.06-0.35$ & 2.80 & 0.008 \\
\hline
\end{tabular}




\begin{tabular}{|llllll|}
\hline & \multicolumn{5}{l}{ Resilience to SDQ Total Difficulties Resilience Factors \& Demographic Variables } \\
\hline Self Esteem: Global Self-Worth & $\mathbf{0 . 0 8}$ & $\mathbf{0 . 0 3}$ & $\mathbf{0 . 0 1 - 0 . 1 4}$ & $\mathbf{2 . 2 5}$ & $\mathbf{0 . 0 3 3}$ \\
\hline Self Esteem: Scholastic Competence & 0.03 & 0.02 & $-0.02-0.07$ & 1.14 & 0.261 \\
\hline Locus of Control - Internalised & 0.25 & 0.16 & $-0.07-0.57$ & 1.56 & 0.127 \\
\hline Mental Flexibility - Middle tertile & 0.09 & 0.17 & $-0.24-0.43$ & 0.56 & 0.580 \\
\hline Mental Flexibility - Fastest tertile & -0.02 & 0.17 & $-0.36-0.33$ & -0.09 & 0.928 \\
\hline Cognitive Skills & $\mathbf{2 . 7 5}$ & $\mathbf{0 . 8 6}$ & $\mathbf{0 . 9 7 - 4 . 5 3}$ & $\mathbf{3 . 1 8}$ & $\mathbf{0 . 0 0 4}$ \\
\hline Temperament & $\mathbf{0 . 2 8}$ & $\mathbf{0 . 0 2}$ & $\mathbf{0 . 2 4 - 0 . 3 2}$ & $\mathbf{1 3 . 6 4}$ & $<0.001$ \\
\hline Grandparent Attachment - Yes & -0.04 & 0.15 & $-0.33-0.26$ & -0.25 & 0.803 \\
\hline Positive Sibling Relationship & $\mathbf{0 . 0 5}$ & $\mathbf{0 . 0 2}$ & $\mathbf{0 . 0 2 - 0 . 0 9}$ & $\mathbf{2 . 9 0}$ & $\mathbf{0 . 0 0 7}$ \\
\hline School Attendance (<10 days off) & 0.51 & 0.29 & $-0.08-1.10$ & 1.75 & 0.090 \\
\hline School Perception & $\mathbf{0 . 1 3}$ & $\mathbf{0 . 0 2}$ & $\mathbf{0 . 0 9 - 0 . 1 8}$ & $\mathbf{5 . 6 4}$ & $<0.001$ \\
\hline Religious Engagement (Yes) & -0.06 & 0.14 & $-0.34-0.22$ & -0.42 & 0.679 \\
\hline Extracurricular Activities - Yes & $\mathbf{0 . 5 4}$ & $\mathbf{0 . 1 4}$ & $\mathbf{0 . 2 6 - 0 . 8 2}$ & $\mathbf{3 . 8 9}$ & $<0.001$ \\
\hline Supportive Friendships & 0.04 & 0.04 & $-0.05-0.12$ & 0.96 & 0.347 \\
\hline
\end{tabular}

\section{Predictive Validity}

\section{Predicting Depressive Symptoms at 18 years with Resilience at 16 years.}

The predictive validity of resilience when measured using the residuals approach was investigated by comparing the predictive power of the residual measure of resilience with other determinants of psychosocial functioning on depressive symptoms at 18years as measured by the SMFQ. The multivariate linear regression model of the square root of depressive symptoms at 18yrs, measured by the SMFQ, predicted by resilience, ACEs and demographic variables is given in Table 4.

Resilience significantly predicted a reduction in the risk of having depressive symptoms. A one standard deviation increase in resilience at 16 years was associated with a 0.12 reduced risk of having depressive symptoms at 18 years (std. $\beta=-0.12, p<0.001$ ). This is a comparable effect size to the effect of ACEs on depressive symptoms. Experiencing bullying, emotional abuse, parental neighbourhood dissatisfaction or violence between the child and partner in adolescence (11-16 years) was significantly associated with higher levels of depressive symptoms at 18 years (bullying, std. $\beta=0.15$, $p<0.001 ;$ emotional abuse, std. $\beta=0.16, p=0.012$; neighbourhood dissatisfaction, std. $\beta=0.11, p=0.018$; violence between child and partner, $\beta=0.15, p=0.001$ ). In line with previous research on sex differences in adolescent depressive symptoms in ALSPAC (Kwong et al., 2019), and in a Norwegian longitudinal cohort (Lundervold et al., 2013), being female is significantly associated with higher levels of depressive symptoms at 18 years (std. $\beta=0.18, p<0.001$ ), compared with males. No other demographic indicator was significantly associated with depressive symptoms at 18 years. There were no significant two-way interactions between ACEs and resilience. 
Table 5

Multiple linear regression model of depression symptoms, as measured by SMFQ, predicted by resilience, demographic factors and ACEs. Adjusted $\mathrm{R}^{2}=0.174(0.146-0.203)$

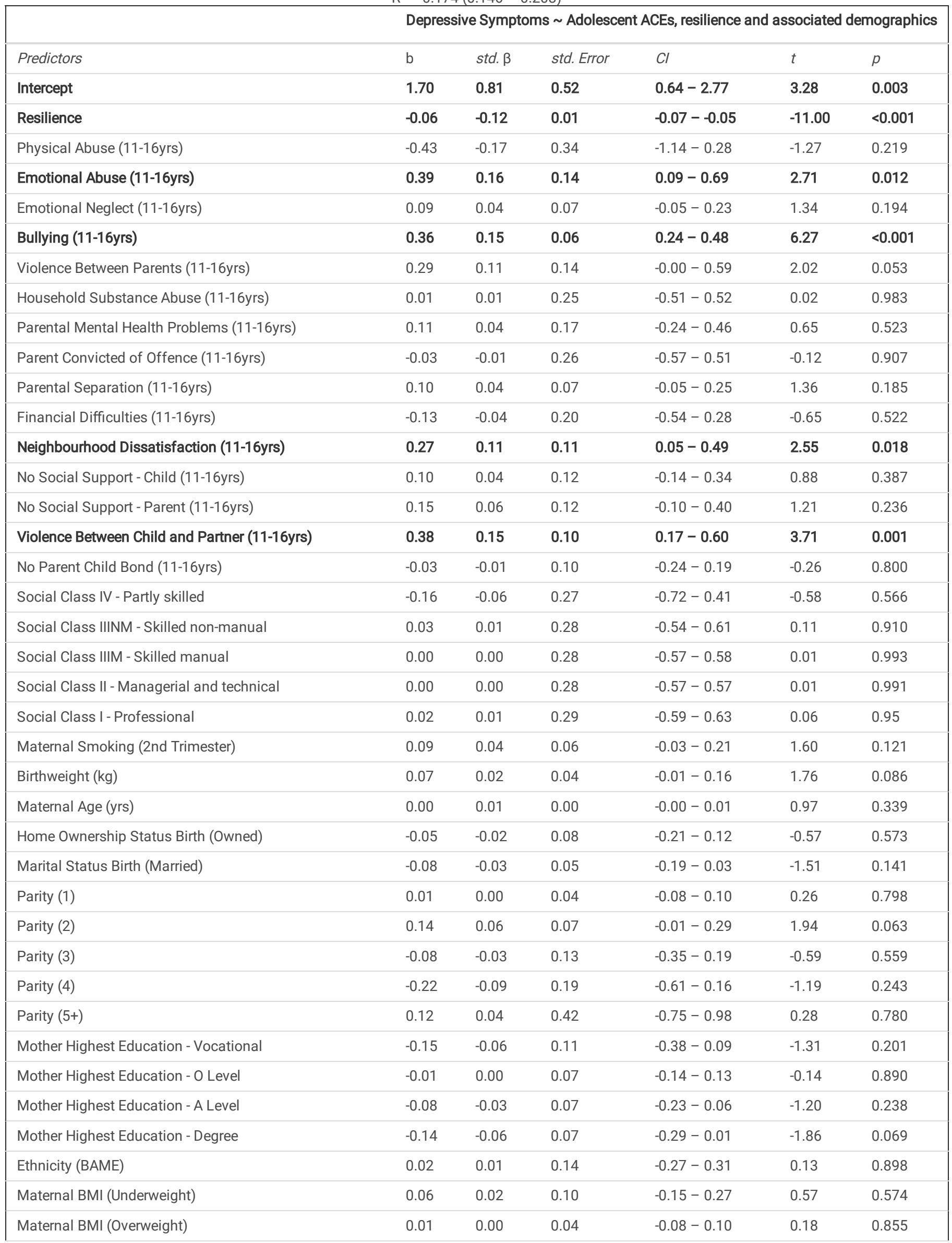




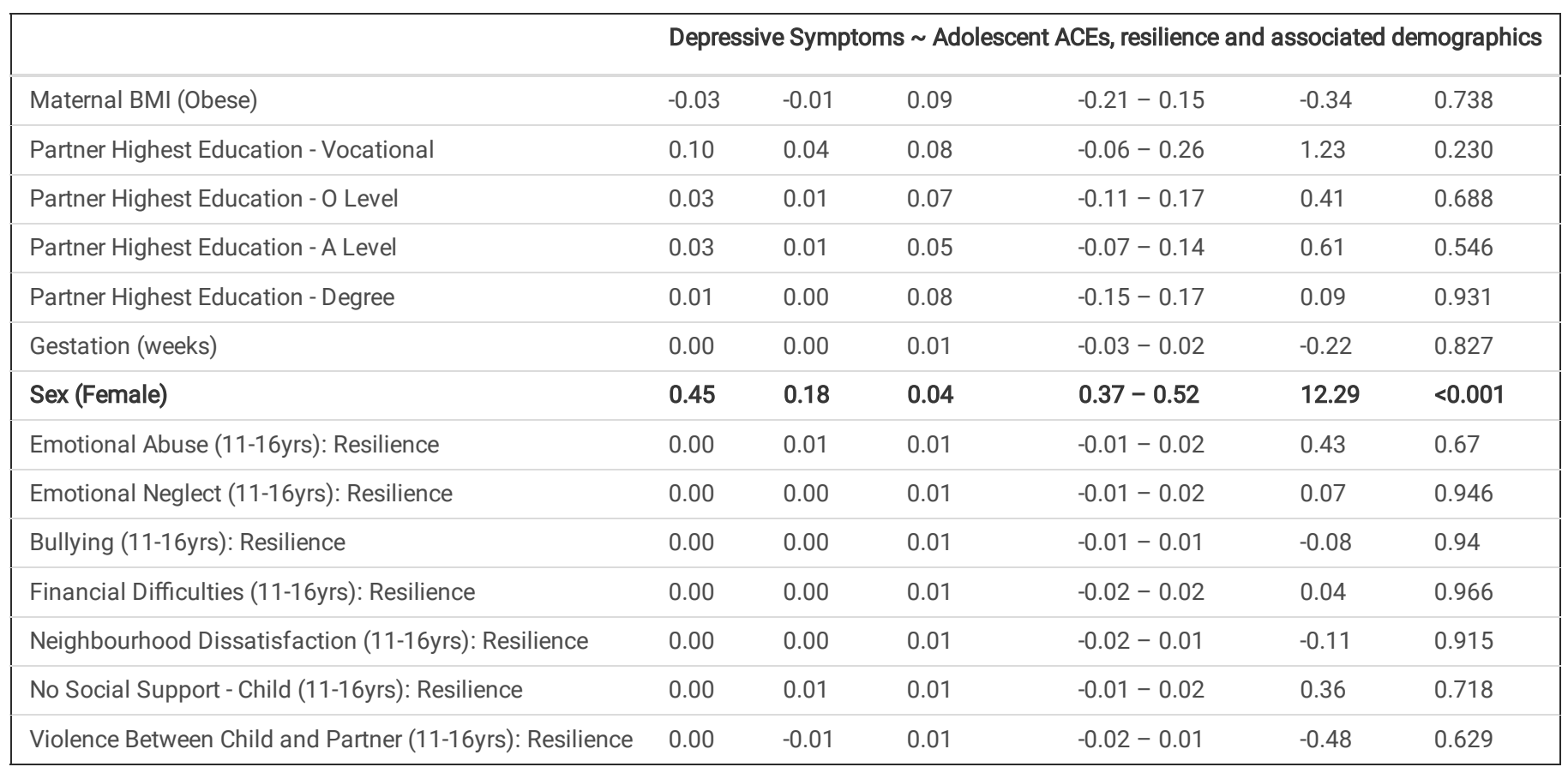

\section{Predictive Validity}

\section{Predicting NEET status at 17 and 23 years with Resilience at 16 years.}

We investigated the predictive validity of resilience when measured using the residuals approach by comparing the predictive power of the residuals measure of resilience with other determinants of psychosocial functioning on NEET status at 17 and 23 years. Resilience to SDQ difficulties at 16 years significantly reduces the risk of having NEET status at 17 years and 23 years. The results are summarised in Table 5.

The likelihood of having NEET status at 17 years is $7 \%$ lower for one SD increase of resilience $(\mathrm{OR}=0.92,95 \% \mathrm{Cl}=0.89-0.97)$. A mother having one or two previous child(ren) increases the likelihood of her children's NEET status by $56 \%(\mathrm{OR}=1.56, \mathrm{Cl}=1.05-2.32)$ or $69 \%(\mathrm{OR}=1.69, \mathrm{Cl}=1.05-2.73)$ respectively. Being born into a home owned by your parents reduces the likelihood of NEET status at 17 by $44 \%(\mathrm{OR}=0.56, \mathrm{Cl}=0.33-0.94)$. For every one year increase in maternal age at birth, the likelihood of having NEET status at 17 is reduced by $5 \%(O R=0.95, \mathrm{Cl}=0.91-0.99)$. Paternal or partner's highest educational qualification of O level $(\mathrm{OR}=0.57, \mathrm{Cl}=0.36-0.92)$ reduced the likelihood of having NEET status at 17 by $43 \%$, when compared to having no or CSA education. In terms of ACEs, experiencing emotional neglect in adolescence was the only ACE with a significant association, with an increase in the likelihood of NEET status at 17 years $(\mathrm{OR}=1.95, \mathrm{Cl}=1.24-3.06)$. Again, there were no significant interactions between ACEs and resilience. 
Table 6

Multiple logistic regression model of NEET status at 17 years predicted by resilience and demographic factors.

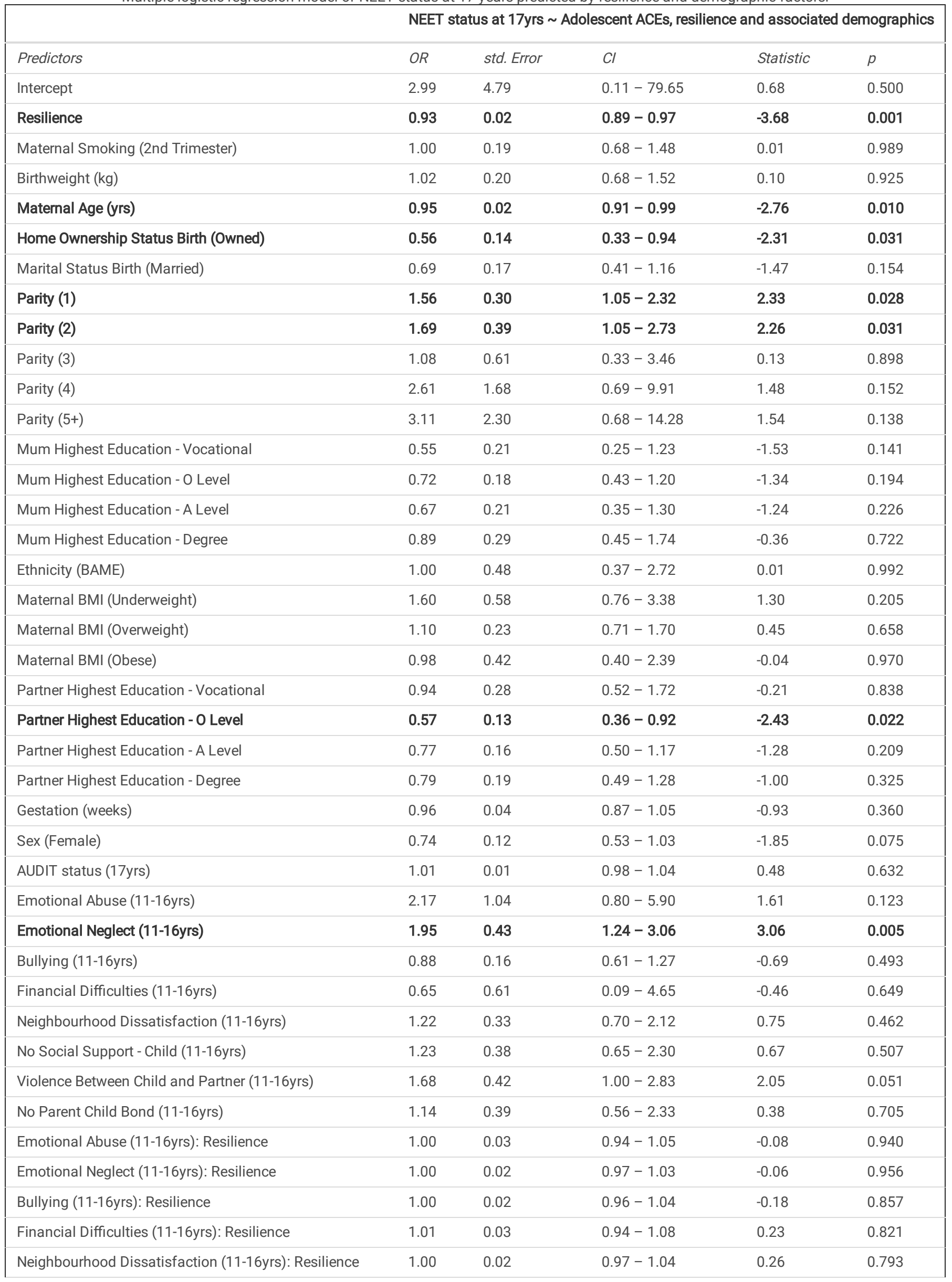

Page 15/25 


\begin{tabular}{|lllll|}
\hline & \multicolumn{4}{c|}{ NEET status at 17yrs Adolescent ACEs, resilience and associated demographics } \\
\hline No Social Support - Child (11-16yrs): Resilience & 0.99 & 0.02 & $0.94-1.04$ & -0.50 \\
\hline Violence Between Child and Partner (11-16yrs): Resilience & 1.00 & 0.02 & $0.97-1.04$ & 0.21 \\
\hline No Parent Child Bond (11-16yrs): Resilience & 1.00 & 0.02 & $0.96-1.04$ & 0.11 \\
\hline
\end{tabular}

The effect of resilience is enduring as the likelihood of having NEET status at 23 years was significantly reduced for each unit increase of resilience (OR = $0.92,95 \% \mathrm{Cl}=0.89-0.95)$. Being NEET at 17 is the strongest predictor of NEET at 23 , increasing the likelihood just over two times $(\mathrm{OR}=2.05, \mathrm{Cl}=1.51-$ 4.04). Children born into a home owned by their parents reduces the likelihood of NEET status at 23 by $30 \%(O R=0.70, \mathrm{Cl}=0.49-0.99)$. If the mother has $\mathrm{O}$ level as the highest educational qualification, the likelihood of having NEET status at 23 was reduced by $35 \%(\mathrm{OR}=0.65, \mathrm{Cl}=0.44-0.96)$, when compared to having no or CSA education. No other demographic variables in the final model showed a significant effect on NEET status at 23 years

The experience of bullying during adolescence increased the likelihood of having NEET status at 23 by $43 \%(\mathrm{OR}=1.43, \mathrm{Cl}=1.05-1.96)$. Having no social support during adolescence increased the likelihood of having NEET status at 23 years by $80 \%(O R=1.80, C l=1.00-3.24)$. There were no significant interactions between ACEs and resilience. 
Table 7

Multiple logistic regression model of NEET status at 23 years predicted by resilience and demographic factors.

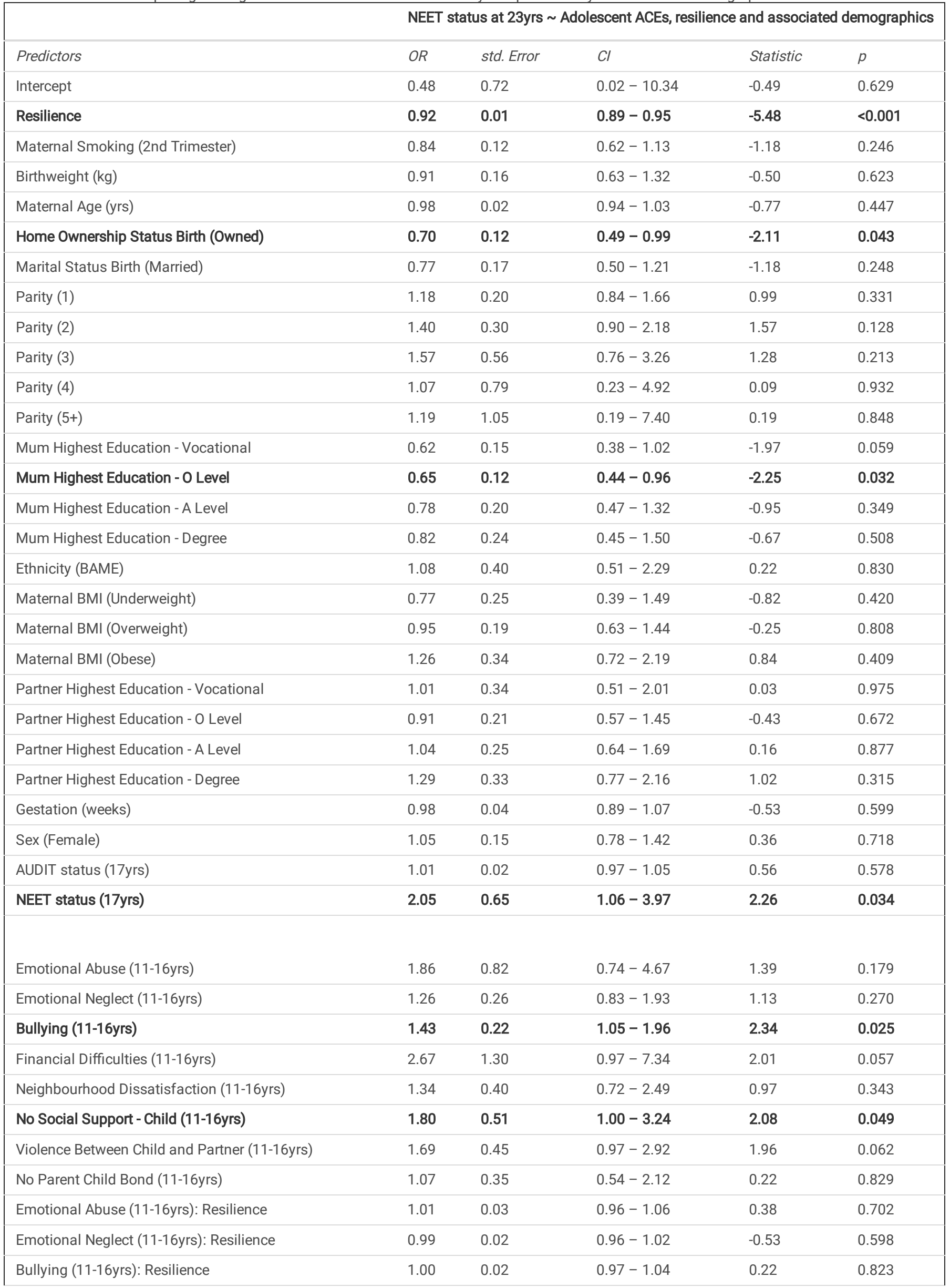




\begin{tabular}{|c|c|c|c|c|c|}
\hline \multirow[b]{2}{*}{ Financial Difficulties (11-16yrs): Resilience } & \multicolumn{5}{|c|}{ NEET status at 23yrs Adolescent ACEs, resilience and associated demographics } \\
\hline & 1.01 & 0.03 & $0.95-1.06$ & 0.21 & 0.834 \\
\hline Neighbourhood Dissatisfaction (11-16yrs): Resilience & 1.00 & 0.02 & $0.97-1.03$ & -0.07 & 0.941 \\
\hline No Social Support - Child (11-16yrs): Resilience & 1.00 & 0.02 & $0.96-1.05$ & 0.07 & 0.946 \\
\hline Violence Between Child and Partner (11-16yrs): Resilience & 1.00 & 0.02 & $0.97-1.03$ & 0.01 & 0.993 \\
\hline No Parent Child Bond (11-16yrs): Resilience & 1.00 & 0.02 & $0.96-1.04$ & 0.11 & 0.914 \\
\hline
\end{tabular}

\section{Discussion}

Although numerous articles have used the residuals approach to measuring resilience (Amstadter et al., 2014; Bowes et al., 2010; Collishaw et al., 2016; Cosco et al., 2018; de Vries et al., 2021; loannidis et al., 2020; Miller-Lewis et al., 2013; Sapouna \& Wolke, 2013; van Harmelen et al., 2017), to our knowledge, no published studies have formally investigated the validity of this approach. Our study has found that the residuals approach to measuring resilience has both construct and predictive validity. Seven resilience factors were associated with higher levels of resilience and their effect on resilience was in line with predictions based on prior research. Resilience significantly predicted a reduction in the risk of having depressive symptoms at 18 years old and predicted a reduction in the likelihood of having NEET status at 17 and 23 years. Surprisingly, no socioeconomic factors were found to be associated with resilience.

\section{Construct Validity}

\section{Individual Resilience Factors}

We sought to assess the validity of this methodology by investigating whether previously identified resilience factors and demographic factors significantly predict resilience when measured by the residuals approach. To demonstrate the validity of our approach, we investigated whether individual factors previously associated with an increase in resilience significantly predicted resilience as measured by the residual approach at 16 years in our ALSPAC sample. Indeed, we found high cognitive skills (at 6 years 9 months), reading comprehension (at 9 years), high global self-worth (8 years), and a less emotional temperament (5 years 9 months) represent intrinsic individual level RFs that continue to exert their positive effects for some length of time. They could also be described as generative, setting positive cascades in place that develop other contributing factors such as coping styles and emotion regulation (Luthar et al., 2006). A less emotional temperament in childhood, described as biologically-based individual differences in reactivity and regulation (Rothbart, 2006), was a significant predictor of resilience at 16 years. These findings are consistent with previous research that has found children with less emotional temperaments are less reactive to stressors, better able to regulate their feelings of sadness and anger, more likely to maintain positive adaptation and activate flexible coping strategies to deal with adversity (Compas et al., 2004; Martinez-Torteya et al., 2009; Olson et al., 2002). The finding that temperament in childhood predicts resilience in adolescence therefore supports the construct validity of the residual measurement of resilience.

High cognitive skills have previously been associated with positive adaptation in the face of adversity (Gartland et al., 2019; Jaffee et al., 2007), predictive of lower levels of psychiatric disorders, lower rates of conduct problems and higher levels of overall functioning (Malcarne et al., 2000). While having a high IQ was just below the significance threshold in our model predicting resilience, high cognitive skills and high reading comprehension significantly predicted resilience. Having well-developed verbal cognitive abilities could allow children to use verbal strategies to mediate conflict, leading to more circumstanceappropriate behavioural choices and a larger range of coping strategies (Buckner et al., 2003). In addition to cognitive skills, high global self-worth at 8 years was associated with higher levels of resilience at 16 years. Self-worth is an intrapersonal characteristic that has been previously reported to impact an individual's potential for resilience (Davey et al., 2003; Reyes, 2008). Individuals with high self-worth have high amounts of self-respect, and have positive feelings about themselves, their environment and their ability to deal with life's challenges, focussing on their strengths (Rutter, 1989; Werner, 1994). Higher self-worth has been linked to positive cognitive reappraisal (Schwerdtfeger et al., 2019), an underlying mechanism that protects against stressors and mediates RFs via cognitive processes (Kalisch et al., 2015).

\section{Family Resilience Factors}

In terms of family resilience factors, previous studies have had broad support for high quality caregiver relationships and stable family environments (Afifi \& MacMillan, 2011; Gartland et al., 2019; Haskett et al., 2006). We found an association between positive sibling relationship and resilience. High-quality sibling relationships are a unique context which can have a direct impact on one another's socioemotional development, behaviour and adjustment, relevant to resilience (Dirks et al., 2015; McHale et al., 2012). It is interesting to note that women who have had one or three previous children, and two just below the threshold, was also significantly associated with an increase in resilience in their offspring, which may suggest that having siblings increases resilience in adolescence. This enduring association between multiparous (giving birth previously) and resilience could also be related to the extensive physiological, hormonal, and emotional changes experienced by the mother during pregnancy and the postpartum period. The hormonal fluctuations required for the maintenance of pregnancy are unmatched by any other neuroendocrine events in a healthy female's lifetime (Brunton \& Russell, 2010), with dramatic changes also evident in metabolic, immune, cardiovascular, pulmonary, haematological and neurobiological systems (Deems \& Leuner, 2020). Despite parity being commonly included as a covariate in studies of psychological outcomes of children of multiparous women, the origins of parity-associated differences in these outcomes remain poorly defined. It is possible that the observed differences reflecting effects of prior pregnancy on adaptation to subsequent pregnancies/children. However, well-documented increased activation of the Hypothalamic-pituitary-adrenal (HPA) axis (e.g.(Conde \& Figueiredo, 2014)) and sympathetic nervous system (DiPietro et al., 2005) in pregnancy is consistent with well-described physiological responses to psychological stress, supporting the role of psychological factors (Gillespie et al., 2018). Maternal mental health problems are also less common following a multiparous pregnancy (e.g. (Iwata et al., 2016)). Specifically related to resilience, parity has been associated with a modification in disease risk and progression of multiple sclerosis, 
depression, stroke and Alzheimer's disease in the mother (Deems \& Leuner, 2020). Disentangling the mechanisms by which maternal parity is associated with adolescent resilience warrants further investigations.

We found little evidence in support of the relationship between grandparent attachment and resilience, perhaps due to the maternal self-reports used in ALSPAC. Although there is some evidence of detection bias, there is evidence of intergenerational transmission of child abuse (Widom et al., 2015). An abusive parent may have been subjected to abuse by their own parent, hence the limited evidence for grandparents exerting a positive influence and predicting resilience. Our construct of the maternal parenting score within ALSPAC had such high missingness (>80\%) that it had to be removed from the missing data analysis. Future research would benefit from including some measure of positive parental engagement as a protective factor.

\section{Community Resilience Factors}

Within our framework of community level resilience factors, factors relating to school, including positive opinion of school and regular participation in extracurricular activities were associated with higher levels of resilience. These extrinsic school-based factors are in keeping with the dynamic model of resilience, which conceptualizes resilience not as an individual trait but a process resulting from interactions across the life span, dependent upon context and resources (Cicchetti \& Blender, 2006; Kalisch et al., 2017; Rutter, 2012). Ungar (Ungar, 2015) proposes that when stressors are particularly high, environmental factors become more critical for a person's resilience than individual characteristics or cognitions.

\section{Lack of Association between Socioeconomic Factors and Resilience}

None of the socioeconomic factors were significantly associated with resilience. This is a somewhat surprising result given that health is well established to be socioeconomically stratified, with those in socioeconomically disadvantaged groups at a greater risk of negative physical and psychological outcomes when compared with socioeconomically advantaged groups (Marmot, 2005). Adult socioeconomic advantage has been previously associated with adult resilience, measured at $60-64$ years using the residuals methodology (Cosco et al., 2018), yet there is scant evidence of childhood socioeconomic advantage being associated with adolescent resilience. If socioeconomic disadvantage is a major determinant of health, then emotional and cognitive responses to this inequality are of crucial importance and it is likely that the residuals approach is capturing these responses, rather than capturing socioeconomic dis/advantage itself. Future research using the same methodological techniques for measuring resilience could assess whether child socioeconomic disadvantage followed by adult socioeconomic advantage (i.e., upward intergenerational social mobility) is associated with greater resilience. This would support the theory of 'steeling' i.e., developing resilience through exposure to mild aversity in early life, which is supported by positive results in animal models (e.g. (Lyons et al., 2009)) yet has limited results in humans (Rutter, 2012).

\section{Implications for Interventions}

Our study provides support for construct validity of the residuals approach to measuring resilience and suggests some key areas that have important implications for policy, practice, and future work. We note that while the results of this study may be informative for population-level or structural policies, we are not individualising the problem or suggesting to place the onus on individuals to act. All of our recommendations for targeted levels of intervention are within the structural social context in which the children are exposed to ACEs (see (Kelly-Irving \& Delpierre, 2019), for further discussion). While many of the individual RFs we measured are not easily modifiable, there is scope for intervention researchers to have success in enhancing language development (GoldinMeadow et al., 2014), cognitive and social-emotional development (Schonert-Reichl et al., 2015) and global self-worth through physical activity (Haugen et al., 2011). Intervening in sibling interactions may be useful to encourage high-quality sibling relationships, with two prevention programs already in place in the US (More Fun with Sisters and Brothers (Kennedy \& Kramer, 2008) and Siblings are Special (Feinberg et al., 2013)). At the community level, our study suggests the school environment is the most important area for policy to focus on and, given that the key protective factors in our study were identified between 5 and 14 years, individuals may particularly benefit from interventions in primary school, particularly school-based strategies that offer a range of extracurricular activities and enable children to feel more positive about school. Policy could target areas that encapsulate multiple protective factors and their intertwined relationships together. For example, the link between physical activity, global self-worth and adaptive cognitive reappraisal (Haugen et al., 2011; PerchtoldStefan et al., 2020) could benefit from extracurricular sports programs.

\section{Predictive Validity - Depressive Symptoms}

We investigated the predictive validity of resilience by comparing the predictive power of the residuals method of quantifying resilience with other determinants of psychosocial functioning on two outcomes. Adjusting for ACEs and other sociodemographic factors associated with increased depressive symptoms, resilience was significantly associated with reduced depressive symptoms at age 18 [the first outcome in the predictive validity analyses, (std. $\beta=$ $-0.12, p<0.001)$. These results strongly support the predictive validity of the residuals method of measuring resilience. The identification of resilience as a specific protective factor associated with lower reports of depressive symptoms can inform the development of prevention and treatment interventions for depression. Specifically, the strategies mentioned above promoting resilience in all children, not just those exposed to ACEs, would be beneficial. Additionally, measuring an adolescent's resilience to SDQ at 16 may be highly informative. Individual differences in resilience scores may have consequences for tailoring prevention interventions for psychiatric disorders. Further research is needed to explore to what extent this measure of resilience has predictive value for prevention and/or clinical intervention.

\section{Predictive Validity - NEET status}

In the second predictive validity analysis, resilience also predicted a reduced likelihood of NEET status at both 17 years $(\mathrm{OR}=0.93,95 \% \mathrm{Cl}=0.89-0.97)$ and 23 years $(\mathrm{OR}=0.92,95 \% \mathrm{Cl}=0.89-0.95)$. While these are modest effect sizes, they are in line with effect sizes previously associated with the predictive validity of self-assessed resilience (Campbell-Sills et al., 2018) and the social competence resilience factor of the resilience scale for adolescents (Hjemdal et 
al., 2007). The continued stable effect of resilience on reduced likelihood of NEET status from 17 to 23 shows that the predictive validity of resilience is enduring. These results indicate that resilience has value as a predictor of both depressive symptoms and risk of NEET status.

\section{Research and Clinical Implications}

There are research and clinical implications that can be derived from this study. First, the residuals approach to measuring resilience has both construct and predictive validity: it is a measure of the current resilience of an individual, where resilient functioning refers to better mental wellbeing compared to other individuals with similar ACE exposures. Accordingly, resilience researchers can benefit from this measurement of resilience to determine specific resilience factors at the individual, family and community level that are associated with higher levels of resilience. In addition, this measure of resilience can be used as an independent variable to predict various outcome variables such as psychosocial outcomes and overall functioning.

The greatest strength of this measurement of resilience is its ability to be derived from a simpler computational framework that does not require specialised latent variable modelling software, which therefore supports the widespread application of this method. Because this measure is data-driven, it is a measure of the current resilience of an individual, where resilient functioning refers to better mental wellbeing compared to other individuals with similar ACE exposures. The derived measure is influenced by the specific variables used and provides an individual operationalisation of resilience that is relatively simple to compute.

The novelty of this method of quantifying resilience is not that it demonstrates the protective effects of resilience factors. Extensive previous research on resilience factors have shown the positive influence of resilience on important outcomes (Johnson \& Wiechelt, 2004; Khambati et al., 2018). Instead, its originality and significance lie in its ability to advance two key research areas that cannot be adequately studied using other measures: 1) mechanisms of resilience and 2) efficacy of interventions designed to increase resilience.

First, the mechanisms underlying the protective effects of resilience are best examined with a quantitative, individual-specific variable that represents the sum of the resilience construct. Proxy measures may represent one small aspect of an individual's total resilience, which includes a vast array of life experiences or adversity exposure but are difficult to measure. Extracting a quantitative measure of resilience that is not rooted in any one definition or measured by one static tool, is a step towards identifying underlying general resilience mechanisms (loannidis et al., 2020; Kalisch et al., 2015).

Second, this quantitative measure of resilience can be measured longitudinally and used as an ongoing measure of change through therapeutic processes. The ability to assess an individual's resilience at the outset of intervention provides a beneficial starting point for strength-based, individual focused care. Extracting a measure of resilience that is sensitive to change can better inform these potential interventions. Similarly, by quantifying resilience at multiple time points, one can characterise individual differences in the variation of resilience and ascertain the impact of resilience factors at the individual, family and community level at varying timepoints across the life course. Future studies are needed to explore this.

\section{Limitations}

There are some limitations in the present study that must be acknowledged. First, as with most longitudinal cohorts, there was attrition in all outcomes. Whilst we attempted to minimize the impact of this using multiple imputation with chained equations, this approach cannot remove bias completely. Secondly, our dataset of SDQ outcomes was derived from maternal reports but the parents may underestimate conduct problems. However, mean ALSPAC scores are similar to national levels (Meltzer et al., 2003). Thirdly, the results found here may be unique to the ALSPAC cohort, a cohort that is very white, with a higher proportion of married mothers who own their own home than the rest of the general population. We therefore need to expand and diversify the sample to allow for these results to be translatable at the population level. To increase the reliability of this measure of resilience, we propose a replication study in a different dataset. Finally, the correlational design cannot determine causal relations, and prospective or experimental studies are needed.

\section{Conclusions}

In sum, this study has shown the construct and predictive validity of quantifying resilience as residual variance in a psychosocial outcome. It has the potential to advance research into the mechanisms and modifiability of resilience. A key next step in applying this method is to learn how a residual resilience variable interacts with stressors over time.

\section{Abbreviations}

ACE - Adverse Childhood Experiences

ALSPAC - Avon Longitudinal Study of Parents and Children

AUDIT - Alcohol Use Disorders Identification Test

BAME - Black and Ethnic Minority

CSA - Compulsory School Age

MAR - Missing at Random

MI - Multiple Imputation

NEET - Not in Employment, Education or Training 
RF - Resilience factors

SDQ - Strength and Difficulties Questionnaire

SMFQ - Short Moods and Feelings Questionnaire

\section{Declarations}

\section{Ethics Approval and Consent to Participate}

Ethical approval for the study was obtained from the ALSPAC Ethics and Law Committee and the Local Research Ethics Committees. Informed consent for the use of data collected via questionnaires and clinics was obtained from participants following the recommendations of the ALSPAC Ethics and Law Committee at the time.

\section{Consent for Publication}

Not applicable

\section{Availability of Data and Materials}

The ALSPAC dataset is available to all researchers on application to ALSPAC data and samples. Please note that the study website contains details of all the data that is available through a fully searchable data dictionary and variable search tool: http://www.bristol.ac.uk/alspac/researchers/our-data/

\section{Competing Interests}

The authors declare they have no competing interests.

\section{Author's Contributions}

SC and TC conceived the initial study idea and design. TC and SC provided input on the methodology for analysing the data. SC did the main data analyses and wrote the manuscript. All authors (i) provided substantial contributions to the conception or design of the work, or the acquisition, analysis, or interpretation of data for the work, (ii) revised the manuscript for important intellectual content, (iii) approved the final version, and (iv) agreed to be accountable for all aspects of the work. The corresponding author had full access to all the data in the study and had final responsibility for the decision to submit for publication. The author(s) read and approved the final manuscript.

\section{Funding}

The UK Medical Research Council and Wellcome (Grant ref: 217065/Z/19/Z) and the University of Bristol provide core support for ALSPAC. This publication is the work of the authors and Stephanie Cahill will serve as guarantors for the contents of this paper. A comprehensive list of grants funding is available on the ALSPAC website (http://www.bristol.ac.uk/alspac/external/documents/grant-acknowledgements.pdf).

SC is supported by ESRC grant ES/P000347/1 'Soc-B (Social-Biological) Centre for Doctoral Training: UCL-Manchester-Essex Consortium'.

\section{Acknowledgements}

We are extremely grateful to all the families who took part in this study, the midwives for their help in recruiting them, and the whole ALSPAC team, which includes interviewers, computer and laboratory technicians, clerical workers, research scientists, volunteers, managers, receptionists and nurses.

We would like to thank Dr Nick Shryane and Dr Ola Demkowicz of the University of Manchester for their helpful comments on a draft of this manuscript.

\section{References}

1. Afifi, T. O., \& MacMillan, H. L. (2011). Resilience following Child Maltreatment: A Review of Protective Factors. The Canadian Journal of Psychiatry, 56(5), 266-272. doi:10.1177/070674371105600505

2. Amstadter, A. B., Myers, J. M., \& Kendler, K. S. (2014). Psychiatric resilience: longitudinal twin study. British Journal of Psychiatry, 205(4), $275-280$. doi:10.1192/bjp.bp.113.130906

3. Angold, A., Costello, E. J., Messer, S. C., \& Pickles, A. (1995). Development of a short questionnaire for use in epidemiological studies of depression in children and adolescents. 5, 237-249.

4. Angold, A., Erkanli, A., Silberg, J., Eaves, L., \& Costello, E. J. (2002). Depression scale scores in 8-17-year-olds: effects of age and gender. Journal of Child Psychology and Psychiatry, 43(8), 1052-1063.

5. Anthony, E. J., \& Cohler, B. J. (1987). The invulnerable child: Guilford Press.

6. Bellis, M. A., Lowey, H., Leckenby, N., Hughes, K., \& Harrison, D. (2013). Adverse childhood experiences: retrospective study to determine their impact on adult health behaviours and health outcomes in a UK population. Journal of Public Health, 36(1), 81-91. doi:10.1093/pubmed/fdt038

7. Bennett, S., Farrington, D. P., \& Huesmann, L. R. (2005). Explaining gender differences in crime and violence: The importance of social cognitive skills. Aggression and violent behavior, 10(3), 263-288. 
8. Bolger, K. E., \& Patterson, C. J. (2001). Pathways from child maltreatment to internalizing problems: Perceptions of control as mediators and moderators. Development and Psychopathology, 13(4), 913-940.

9. Bonanno, G. A. (2005). Resilience in the Face of Potential Trauma. Current directions in psychological science, 14(3), 135-138. doi:10.1111/j.09637214.2005.00347.x

10. Bowes, L., Maughan, B., Caspi, A., Moffitt, T. E., \& Arseneault, L. (2010). Families promote emotional and behavioural resilience to bullying: evidence of an environmental effect. Journal of Child Psychology and Psychiatry, 51(7), 809-817. doi:10.1111/j.1469-7610.2010.02216.x

11. Boyd, A., Golding, J., Macleod, J., Lawlor, D. A., Fraser, A., Henderson, J., . . Davey Smith, G. (2013). Cohort Profile: the 'children of the 90s'-the index offspring of the Avon Longitudinal Study of Parents and Children. International journal of epidemiology, 42(1), 111-127. doi:10.1093/ije/dys064

12. Brunton, P. J., \& Russell, J. A. (2010). Endocrine induced changes in brain function during pregnancy. Brain Research, 1364, $198-215$. doi:https://doi.org/10.1016/j.brainres.2010.09.062

13. Buckner, J. C., Mezzacappa, E., \& Beardslee, W. R. (2003). Characteristics of resilient youths living in poverty: the role of self-regulatory processes. Development and Psychopathology, 15(1), 139-162. doi:10.1017/s0954579403000087

14. Buuren, S. v., \& Groothuis-Oudshoorn, K. (2010). mice: Multivariate imputation by chained equations in R. Journal of statistical software, 1-68.

15. Campbell-Sills, L., Kessler, R. C., Ursano, R. J., Sun, X., Taylor, C. T., Heeringa, S. G., . . Stein, M. B. (2018). Predictive validity and correlates of selfassessed resilience among US Army soldiers. Depression and Anxiety, 35(2), 122-131.

16. Cicchetti, D., \& Blender, J. A. (2006). A multiple-levels-of-analysis perspective on resilience: implications for the developing brain, neural plasticity, and preventive interventions. Annals of the New York Academy of Sciences, 1094, 248-258. Retrieved from http://ovidsp.ovid.com/ovidweb.cgi? $\mathrm{T}=\mathrm{JS} \& \mathrm{PAGE}=$ reference\&D$=$ med $5 \& \mathrm{NEWS}=\mathrm{N} \& \mathrm{AN}=17347356$

17. Collishaw, S., Hammerton, G., Mahedy, L., Sellers, R., Owen, M. J., Craddock, N., . . Thapar, A. (2016). Mental health resilience in the adolescent offspring of parents with depression: a prospective longitudinal study. The Lancet Psychiatry, 3(1), 49-57. doi:10.1016/s2215-0366(15)00358-2

18. Compas, B. E., Connor-Smith, J., \& Jaser, S. S. (2004). Temperament, Stress Reactivity, and Coping: Implications for Depression in Childhood and Adolescence. Journal of Clinical Child \& Adolescent Psychology, 33(1), 21-31. doi:10.1207/S15374424JCCP3301_3

19. Conde, A., \& Figueiredo, B. (2014). 24-h urinary free cortisol from mid-pregnancy to 3-months postpartum: Gender and parity differences and effects. Psychoneuroendocrinology, 50, 264-273.

20. Cosco, T. D., Cooper, R., Kuh, D., \& Stafford, M. (2018). Socioeconomic inequalities in resilience and vulnerability among older adults: a population-based birth cohort analysis. International Psychogeriatrics, 30(5), 695-703. doi:10.1017/S1041610217002198

21. Cronbach, L. J., \& Meehl, P. E. (1955). Construct validity in psychological tests. Psychological Bulletin, 52(4), 281.

22. Crouch, E., Probst, J. C., Radcliff, E., Bennett, K. J., \& McKinney, S. H. (2019). Prevalence of adverse childhood experiences (ACEs) among US children. Child abuse \& neglect, 92, 209-218. doi:https://doi.org/10.1016/j.chiabu.2019.04.010

23. Crush, E., Arseneault, L., Moffitt, T. E., Danese, A., Caspi, A., Jaffee, S. R., . . Fisher, H. L. (2018). Protective factors for psychotic experiences amongst adolescents exposed to multiple forms of victimization. Journal of Psychiatric Research, 104, 32-38. doi:10.1016/j.jpsychires.2018.06.011

24. Davey, M., Eaker, D. G., \& Walters, L. H. (2003). Resilience processes in adolescents: Personality profiles, self-worth, and coping. Journal of adolescent research, 18(4), 347-362.

25. de Vries, L. P., Baselmans, B. M. L., Luykx, J. J., de Zeeuw, E. L., Minică, C. C., de Geus, E. J. C., . . Bartels, M. (2021). Genetic evidence for a large overlap and potential bidirectional causal effects between resilience and well-being. Neurobiology Of Stress, 14, 100315.

doi:https://doi.org/10.1016/j.ynstr.2021.100315

26. Deems, N. P., \& Leuner, B. (2020). Pregnancy, postpartum and parity: Resilience and vulnerability in brain health and disease. Frontiers In Neuroendocrinology, 57, 100820. doi:https://doi.org/10.1016/j.yfrne.2020.100820

27. Department for Education, T. (2013). NEET Statistics - Quarterly Brief - October to December 2013. England

28. DiPietro, J. A., Costigan, K. A., \& Gurewitsch, E. D. (2005). Maternal psychophysiological change during the second half of gestation. Biological Psychology, 69(1), 23-38.

29. Dirks, M. A., Persram, R., Recchia, H. E., \& Howe, N. (2015). Sibling relationships as sources of risk and resilience in the development and maintenance of internalizing and externalizing problems during childhood and adolescence. Clinical psychology review, 42, 145-155. doi:https://doi.org/10.1016/j.cpr.2015.07.003

30. Feinberg, M. E., Solmeyer, A. R., Hostetler, M. L., Sakuma, K.-L., Jones, D., \& McHale, S. M. (2013). Siblings Are Special: Initial Test of a New Approach for Preventing Youth Behavior Problems. Journal of Adolescent Health, 53(2), 166-173. doi:10.1016/j.jadohealth.2012.10.004

31. Felitti, V. J., Anda, R. F., Nordenberg, D., Williamson, D. F., Spitz, A. M., Edwards, V., \& Marks, J. S. (1998). Relationship of childhood abuse and household dysfunction to many of the leading causes of death in adults: The Adverse Childhood Experiences (ACE) Study. American journal of preventive medicine, 14(4), 245-258.

32. Fraser, A., Macdonald-Wallis, C., Tilling, K., Boyd, A., Golding, J., Davey Smith, G., ... Ness, A. (2012). Cohort profile: the Avon Longitudinal Study of Parents and Children: ALSPAC mothers cohort. International journal of epidemiology, 42(1), 97-110.

33. Fritz, J., de Graaff, A. M., Caisley, H., Van Harmelen, A.-L., \& Wilkinson, P. O. (2018). A Systematic Review of Amenable Resilience Factors that Moderate and/or Mediate the Relationship between Childhood Adversity and Mental Health in Young People. Frontiers in psychiatry, 9.

34. Gartland, D., Riggs, E., Muyeen, S., Giallo, R., Afifi, T. O., MacMillan, H., . . Brown, S. J. (2019). What factors are associated with resilient outcomes in children exposed to social adversity? A systematic review. BMJ open, 9(4), e024870-e024870. doi:10.1136/bmjopen-2018-024870

Page $22 / 25$ 
35. Gillespie, S. L., Mitchell, A. M., Kowalsky, J. M., \& Christian, L. M. (2018). Maternal parity and perinatal cortisol adaptation: The role of pregnancy-specific distress and implications for postpartum mood. Psychoneuroendocrinology, 97, 86-93. doi:https://doi.org/10.1016/j.psyneuen.2018.07.008

36. Goldin-Meadow, S., Levine, S. C., Hedges, L. V., Huttenlocher, J., Raudenbush, S. W., \& Small, S. L. (2014). New evidence about language and cognitive development based on a longitudinal study: hypotheses for intervention. American Psychologist, 69(6), 588.

37. Goodman, R. (1997). The Strengths and Difficulties Questionnaire: a research note. Journal of Child Psychology and Psychiatry, 38(5), $581-586$.

38. Graber, R., Turner, R., \& Madill, A. (2016). Best friends and better coping: Facilitating psychological resilience through boys' and girls' closest friendships. British Journal of Psychology, 107(2), 338-358. doi:https://doi.org/10.1111/bjop.12135

39. Harpur, L. J., Polek, E., \& van Harmelen, A.-L. (2015). The role of timing of maltreatment and child intelligence in pathways to low symptoms of depression and anxiety in adolescence. Child abuse \& neglect, 47, 24-37. doi:https://doi.org/10.1016/j.chiabu.2015.05.019

40. Haskett, M. E., Nears, K., Sabourin Ward, C., \& McPherson, A. V. (2006). Diversity in adjustment of maltreated children: Factors associated with resilient functioning. Clinical psychology review, 26(6), 796-812. doi:https://doi.org/10.1016/j.cpr.2006.03.005

41. Haugen, T., Säfvenbom, R., \& Ommundsen, Y. (2011). Physical activity and global self-worth: The role of physical self-esteem indices and gender. Mental Health and Physical Activity, 4(2), 49-56. doi:https://doi.org/10.1016/j.mhpa.2011.07.001

42. Herrenkohl, E. C., Herrenkohl, R. C., Rupert, L. J., Egolf, B. P., \& Lutz, J. G. (1995). Risk factors for behavioral dysfunction: The relative impact of maltreatment, SES, physical health problems, cognitive ability, and quality of parent-child interaction. Child abuse \& neglect, 19(2), 191-203. doi:https://doi.org/10.1016/0145-2134(94)00116-C

43. Herrenkohl, T. I., Tajima, E. A., Whitney, S. D., \& Huang, B. (2005). Protection against antisocial behavior in children exposed to physically abusive discipline. Journal of Adolescent Health, 36(6), 457-465. doi:https://doi.org/10.1016/j.jadohealth.2003.09.025

44. Hjemdal, O., Aune, T., Reinfjell, T., Stiles, T. C., \& Friborg, O. (2007). Resilience as a predictor of depressive symptoms: a correlational study with young adolescents. Clinical child psychology and psychiatry, 12(1), 91-104.

45. Houtepen, L., Heron, J., Suderman, M., Tilling, K., \& Howe, L. (2018). Adverse childhood experiences in the children of the Avon Longitudinal Study of Parents and Children (ALSPAC) [version 1; referees: 3 approved]. Wellcome Open Research, 3(106). doi:10.12688/wellcomeopenres.14716.1

46. Howe, L. D., Tilling, K., Galobardes, B., \& Lawlor, D. A. (2013). Loss to follow-up in cohort studies: bias in estimates of socioeconomic inequalities. Epidemiology (Cambridge, Mass.), 24(1), 1-9. doi:10.1097/EDE.0b013e31827623b1

47. Hughes, K., Bellis, M. A., Hardcastle, K. A., Sethi, D., Butchart, A., Mikton, C., . . Dunne, M. P. (2017). The effect of multiple adverse childhood experiences on health: a systematic review and meta-analysis. The Lancet Public Health, 2(8), e356-e366.

48. Hughes, K., Lowey, H., Quigg, Z., \& Bellis, M. A. (2016). Relationships between adverse childhood experiences and adult mental well-being: results from an English national household survey. BMC Public Health, 16, 222-222. doi:10.1186/s12889-016-2906-3

49. Ioannidis, K., Askelund, A. D., Kievit, R. A., \& Van Harmelen, A.-L. (2020). The complex neurobiology of resilient functioning after childhood maltreatment. BMC Medicine, 18(1), 1-16.

50. Iwata, H., Mori, E., Sakajo, A., Aoki, K., Maehara, K., \& Tamakoshi, K. (2016). Prevalence of postpartum depressive symptoms during the first 6 months postpartum: association with maternal age and parity. Journal of Affective Disorders, 203, 227-232.

51. Jaffee, S. R., Caspi, A., Moffitt, T. E., Polo-Tomás, M., \& Taylor, A. (2007). Individual, family, and neighborhood factors distinguish resilient from nonresilient maltreated children: A cumulative stressors model. Child abuse \& neglect, 31(3), 231-253. doi:https://doi.org/10.1016/j.chiabu.2006.03.011

52. Johnson, J. L., \& Wiechelt, S. A. (2004). Introduction to the special issue on resilience. Substance Use \& Misuse, 39(5), 657-670. doi:10.1081/JA120034010

53. Kalisch, R., Baker, D. G., Basten, U., Boks, M. P., Bonanno, G. A., Brummelman, E., . . Kleim, B. (2017). The resilience framework as a strategy to combat stress-related disorders. Nature Human Behaviour, 1(11), 784-790. doi:10.1038/s41562-017-0200-8

54. Kalisch, R., Muller, M. B., \& Tuscher, O. (2015). A conceptual framework for the neurobiological study of resilience. The Behavioral And Brain Sciences, 38, e92. doi:10.1017/S0140525X1400082X

55. Kelly-Irving, M., \& Delpierre, C. (2019). A critique of the adverse childhood experiences framework in epidemiology and public health: uses and misuses. Social Policy and Society, 18(3), 445-456.

56. Kennedy, D. E., \& Kramer, L. (2008). Improving emotion regulation and sibling relationship quality: The more fun with sisters and brothers program. Family Relations, 57(5), 567-578.

57. Kessler, R. C., McLaughlin, K. A., Green, J. G., Gruber, M. J., Sampson, N. A., Zaslavsky, A. M., . . Williams, D. R. (2010). Childhood adversities and adult psychopathology in the WHO World Mental Health Surveys. The British journal of psychiatry, 197(5), 378-385. doi:10.1192/bjp.bp.110.080499

58. Khambati, N., Mahedy, L., Heron, J., \& Emond, A. (2018). Educational and emotional health outcomes in adolescence following maltreatment in early childhood: A population-based study of protective factors. Child abuse \& neglect, 81, 343-353. doi:http://dx.doi.org/10.1016/j.chiabu.2018.05.008

59. Kwong, A. S. F., Manley, D., Timpson, N. J., Pearson, R. M., Heron, J., Sallis, H., . . Leckie, G. (2019). Identifying Critical Points of Trajectories of Depressive Symptoms from Childhood to Young Adulthood. Journal Of Youth And Adolescence, 48(4), 815-827. doi:10.1007/s10964-018-0976-5

60. Lacey, R. E., \& Minnis, H. (2020). Practitioner review: twenty years of research with adverse childhood experience scores-advantages, disadvantages and applications to practice. Journal of Child Psychology and Psychiatry, 61(2), 116-130.

61. Little, R. J., \& Rubin, D. B. (2019). Statistical analysis with missing data (Vol. 793): John Wiley \& Sons.

62. Lundervold, A., Breivik, K., Posserud, M.-B., Stormark, K. M., \& Hysing, M. (2013). Symptoms of depression as reported by Norwegian adolescents on the Short Mood and Feelings Questionnaire. Frontiers In Psychology, 4(613). doi:10.3389/fpsyg.2013.00613

Page $23 / 25$ 
63. Luthar, S. S., Sawyer, J. A., \& Brown, P. J. (2006). Conceptual issues in studies of resilience: past, present, and future research. Annals of the New York Academy of Sciences, 1094, 105-115. doi:10.1196/annals.1376.009

64. Lyons, D. M., Parker, K. J., Katz, M., \& Schatzberg, A. F. (2009). Developmental cascades linking stress inoculation, arousal regulation, and resilience. Frontiers In Behavioral Neuroscience, 3, 32.

65. Malcarne, V. L., Hamilton, N. A., Ingram, R. E., \& Taylor, L. (2000). Correlates of distress in children at risk for affective disorder: exploring predictors in the offspring of depressed and nondepressed mothers. J Affect Disord, 59(3), 243-251. doi:10.1016/s0165-0327(99)00155-x

66. Marmot, M. (2005). Social determinants of health inequalities. The Lancet, 365(9464), 1099-1104.

67. Martinez-Torteya, C., Anne Bogat, G., Von Eye, A., \& Levendosky, A. A. (2009). Resilience Among Children Exposed to Domestic Violence: The Role of Risk and Protective Factors. Child development, 80(2), 562-577. doi:https://doi.org/10.1111/j.1467-8624.2009.01279.x

68. Masten, A. (2014). Ordinary magic: Resilience in development. New York: Guilford Press.

69. McHale, S. M., Updegraff, K. A., \& Whiteman, S. D. (2012). Sibling Relationships and Influences in Childhood and Adolescence. Journal of marriage and the family, 74(5), 913-930. doi:10.1111/j.1741-3737.2012.01011.x

70. Meehan, A. J., Baldwin, J. R., Lewis, S. J., MacLeod, J. G., \& Danese, A. (2021). Poor Individual Risk Classification From Adverse Childhood Experiences Screening. American journal of preventive medicine. doi:https://doi.org/10.1016/j.amepre.2021.08.008

71. Meltzer, H., Gatward, R., Goodman, R., \& Ford, T. (2003). Mental health of children and adolescents in Great Britain. International Review of Psychiatry, 15(1-2), 185-187. doi:10.1080/0954026021000046155

72. Miller-Lewis, L. R., Searle, A. K., Sawyer, M. G., Baghurst, P. A., \& Hedley, D. (2013). Resource factors for mental health resilience in early childhood: An analysis with multiple methodologies. Child and adolescent psychiatry and mental health, 7(1), 6. Retrieved from https://www.ncbi.nlm.nih.gov/pmc/articles/PMC3598384/pdf/1753-2000-7-6.pdf

73. Olson, S. L., Bates, J. E., Sandy, J. M., \& Schilling, E. M. (2002). Early developmental precursors of impulsive and inattentive behavior: from infancy to middle childhood. Journal of Child Psycholgy and Psychiatry, 43(4), 435-447. doi:10.1111/1469-7610.00035

74. ONS, O. f. N. S. (2018). Young people not in education, employment or training (NEET).

https://www.ons.gov.uk/employmentandlabourmarket/peoplenotinwork/unemployment/bulletins/youngpeoplenotineducationemploymentortrainingneet/

75. Organisation for Economic Co-operation and Development, T. (2012). NEET rates among youth in countries: Percentage of population aged 15-24. OECD Employment Outlook 2012: OECD Publishing

76. Pargas, R. C. M., Brennan, P. A., Hammen, C., \& Le Brocque, R. (2010). Resilience to Maternal Depression in Young Adulthood. Developmental psychology, 46(4), 805-814. doi:10.1037/a0019817

77. Peck, S. C., Roeser, R. W., Zarrett, N., \& Eccles, J. S. (2008). Exploring the roles of extracurricular activity quantity and quality in the educational resilience of vulnerable adolescents: Variable-and pattern-centered approaches. Journal of Social Issues, 64(1), 135-156.

78. Perchtold-Stefan, C. M., Fink, A., Rominger, C., Weiss, E. M., \& Papousek, I. (2020). More habitual physical activity is linked to the use of specific, more adaptive cognitive reappraisal strategies in dealing with stressful events. Stress and Health, 36(3), 274-286. doi:https://doi.org/10.1002/smi.2929

79. Perkins, D. F., \& Jones, K. R. (2004). Risk behaviors and resiliency within physically abused adolescents. Child abuse \& neglect, $28(5), 547-563$. doi:https://doi.org/10.1016/j.chiabu.2003.12.001

80. Ploubidis, G. B., Benova, L., Grundy, E., Laydon, D., \& DeStavola, B. (2014). Lifelong socio economic position and biomarkers of later life health: Testing the contribution of competing hypotheses. Social science \& medicine, 119, 258-265.

81. Qouta, S., El-Sarraj, E., \& Punamäki, R.-L. (2001). Mental flexibility as resiliency factor among children exposed to political violence. International Journal of Psychology, 36(1), 1-7. doi:10.1080/00207590042000010

82. Reyes, C. J. (2008). Exploring the Relations Among the Nature of the Abuse, Perceived Parental Support, and Child's Self-Concept and Trauma Symptoms Among Sexually Abused Children. Journal of Child Sexual Abuse, 17(1), 51-70. doi:10.1080/10538710701884482

83. Rothbart, M. K., \& Bates, J. E. . (2006). Temperament. In W. L. Damon, R. M., \& Eisenberg, N. (Ed.), Handbook of child psychology: Vol. 3, social, emotional, and personality development (6th ed., Vol. 3, pp. 99-106). New York: NY: Wiley.

84. Rutter, M. (1989). Pathways from childhood to adult life. Journal of Child Psychology and Psychiatry, 30(1), 23-51.

85. Rutter, M. (2012). Resilience as a dynamic concept. Development and Psychopathology, 24(2), 335-344. doi:10.1017/S0954579412000028

86. Sapouna, M., \& Wolke, D. (2013). Resilience to bullying victimization: The role of individual, family and peer characteristics. Child abuse \& neglect, 37(11), 997-1006.

87. Schaefer, L. M., Howell, K. H., Schwartz, L. E., Bottomley, J. S., \& Crossnine, C. B. (2018). A concurrent examination of protective factors associated with resilience and posttraumatic growth following childhood victimization. Child abuse \& neglect. doi:10.1016/j.chiabu.2018.08.019

88. Schechter, J. C., Brennan, P. A., Smith, A. K., Stowe, Z. N., Newport, D. J., \& Johnson, K. C. (2017). Maternal Prenatal Psychological Distress and Preschool Cognitive Functioning: the Protective Role of Positive Parental Engagement. Journal of abnormal child psychology, 45(2), 249-260. doi:10.1007/s10802016-0161-9

89. Schonert-Reichl, K. A., Oberle, E., Lawlor, M. S., Abbott, D., Thomson, K., Oberlander, T. F., \& Diamond, A. (2015). Enhancing cognitive and social-emotional development through a simple-to-administer mindfulness-based school program for elementary school children: A randomized controlled trial [American Psychological Association doi:10.1037/a0038454]. Retrieved

90. Schultze-Lutter, F., Schimmelmann, B. G., \& Schmidt, S. J. (2016). Resilience, risk, mental health and well-being: associations and conceptual differences. European Child and Adolescent Psychiatry, 25(5), 459-466. doi:10.1007/s00787-016-0851-4

Page $24 / 25$ 
91. Schwerdtfeger, A. R., Heene, S., \& Messner, E.-M. (2019). Interoceptive awareness and perceived control moderate the relationship between cognitive reappraisal, self-esteem, and cardiac activity in daily life. International Journal of Psychophysiology, 141, 84-92.

doi:https://doi.org/10.1016/j.jpsycho.2019.04.003

92. Tugade, M. M., \& Fredrickson, B. L. (2004). Resilient individuals use positive emotions to bounce back from negative emotional experiences. Journal of personality and social psychology, 86(2), 320-333. doi:10.1037/0022-3514.86.2.320

93. Turner, N., Joinson, C., Peters, T. J., Wiles, N., \& Lewis, G. (2014). Validity of the Short Mood and Feelings Questionnaire in late adolescence. Psychological assessment, 26(3), 752.

94. Ungar, M. (2015). Practitioner Review: Diagnosing childhood resilience - a systemic approach to the diagnosis of adaptation in adverse social and physical ecologies. Journal of Child Psychology and Psychiatry, 56(1), 4-17. doi:10.1111/jcpp.12306

95. van Harmelen, A. L., Kievit, R. A., loannidis, K., Neufeld, S., Jones, P. B., Bullmore, E., . . Goodyer, I. (2017). Adolescent friendships predict later resilient functioning across psychosocial domains in a healthy community cohort. Psychological Medicine, 47(13), 2312-2322. doi:10.1017/S0033291717000836

96. Werner, E. E. (1994). Overcoming the odds. Journal of developmental and Behavioral Pediatrics.

97. Werner, E. E. (1997). Vulnerable but invincible: high-risk children from birth to adulthood. Acta Paediatrica, 86(S422), $103-105$. doi:https://doi.org/10.1111/j.1651-2227.1997.tb18356.x

98. Werner, E. E., \& Smith, R. S. (2001). Journeys from childhood to midlife: Risk, resilience, and recovery. Cornell University Press.

99. Widom, C. S., Czaja, S. J., \& DuMont, K. A. (2015). Intergenerational transmission of child abuse and neglect: Real or detection bias? Science, 347(6229), 1480-1485. doi:10.1126/science. 1259917

100. Williams, J., \& Nelson-Gardell, D. (2012). Predicting resilience in sexually abused adolescents. Child abuse \& neglect, $36(1), 53-63$. doi:https://doi.org/10.1016/j.chiabu.2011.07.004

101. Zumbo, B. D., \& Chan, E. K. (2014). Reflections on validation practices in the social, behavioral, and health sciences. In Validity and validation in social, behavioral, and health sciences (pp. 321-327): Springer.

\section{Supplementary Figure}

Supplementary Figure 1 is not available with this version.

\section{Figures}

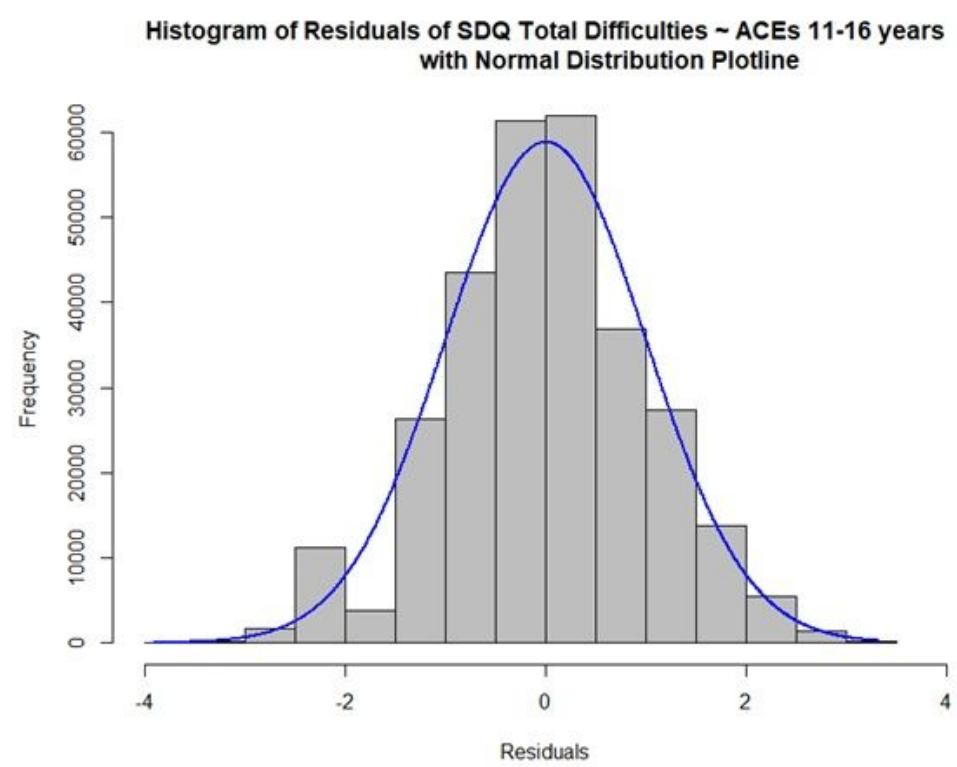

Figure 1

Histogram of the residual variance of the multiple linear regression model: SDQ total difficulties at 16 years $\sim$ ACEs $11-16$ years with normal distribution plotline

\section{Supplementary Files}

This is a list of supplementary files associated with this preprint. Click to download.

- SupplementaryTable1.docx 